\title{
Consistency of returns-to-scale characterizations of production frontiers with respect to model specification
}

\author{
Victor V. Podinovskia,*, Tatiana Bouzdine-Chameeva ${ }^{b}$ \\ ${ }^{a}$ School of Business and Economics, Loughborough University, Loughborough LE11 3TU, UK \\ ${ }^{b}$ KEDGE Business School, 680 cours de la Libération, 33405 Talence Cedex, France
}

\begin{abstract}
Returns-to-scale (RTS) characterizations and the underlying notion of scale elasticity are important characteristics of production frontiers, in both parametric and nonparametric methodologies of efficiency and productivity analysis. In practical applications of these methodologies, the model of technology is often experimented with and modified before it is finalized, which involves, for example, a change of the data set, incorporation, exclusion or aggregation of inputs and outputs, or experimentation with the production assumptions, or axioms, on which the model is based. While it is well-known how such modifications of technology affect the efficiency scores, their effect on the RTS characterization of the production frontier has not been sufficiently explored in the literature. In this paper we obtain several general results that clarify this issue.
\end{abstract}

Keywords: Data envelopment analysis, Production technology, Returns to scale, Scale elasticity

\section{Introduction}

Practical applications of efficiency and productivity analysis, including data envelopment analysis (DEA), often involve experimentation with different specifications of the production technology. The purpose of such experimentations is usually to develop a suitable model of the production process the use of which would produce meaningful results and deliver acceptable discrimination on efficiency between the decision making units (DMUs).

In the methodological framework of DEA, one can choose from a large number of production technologies based on different production assumptions, or axioms (Färe, Grosskopf, \& Lovell, 1985). Depending on the context, such technologies may, for example, incorporate multiple component processes (Imanirad, Cook, \& Zhu, 2013; Podinovski, Olesen, \& Sarrico, 2018), unobserved DMUs (Thanassoulis \& Allen, 1998), additional information in the form of weight restrictions and production trade-offs (Allen, Athanassopoulos, Dyson, \& Thanassoulis, 1997; Podinovski, 2004c; Podinovski \& Bouzdine-Chameeva, 2013), weakly disposable inputs and outputs (Mehdiloo \& Podinovski, 2019), limits on the values of inputs and outputs (Cooper, Pastor, Borras, Aparicio, \& Pastor, 2011) and specific network structures (Kao, 2017; Sahoo, Zhu, Tone, \& Klemen, 2014).

Further modelling options involve the choice of inputs and outputs. For example, a question often arises whether some inputs or outputs should be included or omitted, or if

\footnotetext{
*Corresponding author

Email addresses: v.podinovski@lboro.ac.uk (Victor V. Podinovski), tatiana.chameeva@kedgebs.com (Tatiana Bouzdine-Chameeva)
} 
some of them should be aggregated or disaggregated. An example of the latter is the question of using individual costs as separate inputs or their sum as a single input representing the total cost. On the output side, the choice may concern the use of several products as separate outputs or their aggregate monetary value as a single output. The practical need for aggregation of inputs and outputs, e.g., into total costs and revenue, and understanding its impact, e.g., bias arising in the efficiency assessment, were recently highlighted and discussed as one of the challenges in the context of "big data" by Zelenyuk (2019).

Ideally, when experimenting with different models of technology, we should be aware of the potential effects of changing their specifications on the results of analysis. Such effects concerning the efficiency scores are well known and can be used as a guidance in the process of model specification (see, e.g., Podinovski \& Thanassoulis, 2007). Namely, if we need to improve the efficiency discrimination of the model, we may use a larger technology or reduce the number of inputs and outputs. Conversely, if we are concerned that the assumptions underlying the model are too strong and potentially unrealistic, we may prefer to use weaker and safer assumptions (e.g., by excluding DMUs operating in a potentially different environment or by using more relaxed weight restrictions) resulting in a smaller technology, at the expense of reduced discrimination on efficiency.

In the case of standard constant and variable returns-to-scale (CRS and VRS) DEA models of Charnes, Cooper and Rhodes (1978) and Banker, Charnes and Cooper (1984), ${ }^{1}$ the positive impact of using a larger sample size (resulting in a larger model of technology) and a smaller number of inputs and outputs on the discriminatory power of DEA models was described in the earlier literature (Thanassoulis, Dyson, \& Foster, 1987). Different heuristic rules (rules of thumb) were proposed for a relationship between the sample size and the number of inputs and outputs that should normally be sufficient for obtaining a reasonable discrimination on efficiency - see, e.g., Boussofiane, Dyson and Thanassoulis (1991) and Cooper, Seiford and Tone (2000). The impact of assumptions leading to a larger model of technology on the efficiency scores was illustrated and discussed by Diewert and Mendoza (2007).

The impact of aggregation and disaggregation of inputs and outputs on the efficiency scores in nonparametric models was demonstrated in the earlier works of Nunamaker (1985), Ahn, Charnes, and Cooper (1988) and Thrall (1989). Empirical studies of such impact were conducted by Thomas and Tauer (1994), Tauer and Hanchar (1995) and Tauer (2001). Färe and Zelenyuk (2002) and Färe, Grosskopf and Zelenyuk (2004) obtained theoretical bounds on the bias in efficiency assessment under different conditions on the linear aggregation of inputs and outputs. ${ }^{2}$

The purpose of this paper is to consider how similar modifications of technology affect the returns-to-scale (RTS) characterization of its production frontier. The type of RTS (constant, increasing and decreasing) exhibited by a DMU on the production frontier is defined by the scale elasticity. The latter was originally explored in the case of a single output production function by Johnson (1913) and referred to as the passus coefficient by

\footnotetext{
${ }^{1}$ Equivalent statements of these technologies are also found in the literature on productivity analysis preceding their appearance in the DEA literature - see, e.g., Afriat (1972) and Färe, Grosskopf and Logan (1983).

${ }^{2}$ Another strand of literature is devoted to the effects of aggregation of DMUs into larger units (e.g., branches or divisions to whole companies and further to sectoral levels). For example, Blackorby and Russell (1999) and Briec, Dervaux, and Leleu (2003) consider consistency of efficiency indexes at different levels of aggregation of DMUs. Zelenyuk (2015) studies aggregation of scale efficiency, while the results of Färe and Grosskopf (1985) apply to the aggregation of scale efficiency in the context of cost functions. This type of aggregation of DMUs into larger units is not considered in our paper.
} 
Frisch (1965). The modern notion of scale elasticity for a multiple output production was established by the works of Hanoch (1970), Panzar and Willig (1977) and Starrett (1977).

There exists extensive literature on the evaluation of scale elasticity and RTS in different, parametric and nonparametric, technologies - see, e.g., reviews in Banker, Cooper, Seiford, and Zhu (2011), Sahoo and Tone (2015), and Balk, Färe, and Karagiannis (2015). In particular, Podinovski, Chambers, Atici, and Deineko (2016) develop a universal approach to the evaluation of scale elasticity (among various other marginal characteristics) for production frontiers of arbitrary nonparametric polyhedral technologies.

While the evaluation of RTS has been researched in the literature in depth, relatively few studies have explored potential impacts of the modification of the model of technology on the scale elasticity and RTS characterization. One exception of this is found in Podinovski and Førsund (2010) who investigate the effect of adding additional observed DMUs on the RTS characterization of the production frontier of the VRS technology.

In the current paper we consider this issue in detail assuming only some basic properties of the technology. Specifically, we explore two common scenarios. The first scenario involves the case of one technology being a subset of another. The second scenario is concerned with the aggregation or omission of some inputs and outputs, resulting in a technology with a smaller set of inputs and outputs.

It is worth noting that the focus of our paper is different from the question of stability of RTS characterizations first considered by Seiford and Zhu (1999) and further clarified by Jahanshahloo, Lofti and Zohrehbandian (2005) and Seiford and Zhu (2005). These studies consider the standard VRS model of Banker et al. (1984) and explore the maximum proportional changes of the input and output vectors of the single DMU under the assessment that does not change its RTS type.

In contrast with the existing literature, in our paper we explore the effect of model specification (using a larger technology or omitting or aggregating some inputs or outputs) on the RTS characterization of all DMUs on the production frontier. Also, unlike the existing literature, we are not limited by any particular model of technology. Our results are universally applicable to a variety of different (convex and nonconvex) technologies, under extremely weak production assumptions.

We proceed as follows. In Section 2, we give a brief introduction to the notion of onesided scale elasticities and show their relationship to the RTS characterization of production frontiers. In Section 3, we explore possible changes of the RTS characterization arising from the expansion of the model of technology. In Section 4, we consider the same issue in the case when the technology is obtained by aggregation or omission of some inputs or outputs. Section 5 includes a summary and final remarks. All mathematical proofs are given in Appendix A.

\section{Preliminaries}

Let $\mathcal{T} \subset \mathbb{R}_{+}^{m} \times \mathbb{R}_{+}^{s}$ be a production technology with $m$ inputs and $s$ outputs. Each DMU in $\mathcal{T}$ is stated as $(X, Y)$, where $X \in \mathbb{R}_{+}^{m}$ is a vector of inputs and $Y \in \mathbb{R}_{+}^{s}$ is a vector of outputs. The DMU under the consideration is denoted $\mathrm{DMU}_{o}=\left(X_{o}, Y_{o}\right)$. We assume that $X_{o}$ and $Y_{o}$ are nonzero vectors, although allow some of their component inputs and outputs to be equal to zero.

Technology $\mathcal{T}$ is usually assumed to satisfy certain production axioms that reflect the nature of the underlying production process. To keep our exposition applicable to as wide range of technologies as possible, we generally only assume that technology $\mathcal{T}$ is a closed set and that the set of outputs producible from any vector of inputs is bounded (Färe et al., 
1985). For some specific results, we make an additional explicit assumption that technology $\mathcal{T}$ is convex.

\subsection{Scale elasticity}

The notion of scale elasticity represents the rate at which the vectors of outputs respond to marginal proportional changes of the vectors of inputs, as observed on the production frontier. For a formal definition of this response, and following Starrett (1977) and Førsund (1996), we first consider the output response function defined for any $\mathrm{DMU}_{o}$ in $\mathcal{T}$ :

$$
\bar{\beta}(\alpha)=\max \left\{\beta \mid\left(\alpha X_{o}, \beta Y_{o}\right) \in \mathcal{T}, \beta \in \mathbb{R}\right\} .
$$

The function $\bar{\beta}(\alpha)$ is defined for $\alpha \geq 0$ for which there exists a $\beta$ such that $\left(\alpha X_{o}, \beta Y_{o}\right) \in$ $\mathcal{T}$. The set of all such $\alpha$ (which includes $\alpha=1$ ) is the domain $\Gamma$ of the function $\bar{\beta}(\alpha){ }^{3}$ For each $\alpha \in \Gamma$, the optimal value $\bar{\beta}(\alpha)$ of the maximization program (1) represents the maximum proportion $\beta$ such that the DMU $\left(\alpha X_{o}, \beta Y_{o}\right) \in \mathcal{T}^{4}{ }^{4}$ It is clear that the function $\bar{\beta}(\alpha)$ is a multiplicative analogue of the directional distance function of Chambers, Chung and Färe (1998) defined by the directional vector $g_{Y}=Y_{o}$ (see, e.g., Podinovski et al., 2016).

The notions of scale elasticity and returns to scale are correctly defined for any $\mathrm{DMU}_{o}$ located on the production frontier. This requirement is stated as the following assumption.

Assumption 1. DMU $\left(X_{o}, Y_{o}\right)$ is output radial efficient, i.e.,

$$
\bar{\beta}(1)=\max \left\{\beta \mid\left(X_{o}, \beta Y_{o}\right) \in \mathcal{T}, \beta \in \mathbb{R}\right\}=1 .
$$

If technology $\mathcal{T}$ has a sufficiently smooth production frontier, the function $\bar{\beta}(\alpha)$ is differentiable at $\alpha=1$. In this case, the scale elasticity $\varepsilon\left(X_{o}, Y_{o}\right)$ evaluated at $\mathrm{DMU}_{o}$ is defined as the derivative of the function $\bar{\beta}(\alpha)$ at $\alpha=1$ (Hanoch, 1970; Panzar \& Willig, 1977; Starrett, 1977):

$$
\varepsilon\left(X_{o}, Y_{o}\right)=\bar{\beta}^{\prime}(1)
$$

The definition of scale elasticity (2) is useful in parametric methodologies in which the production frontier of technology $\mathcal{T}$ is defined by the equation $F(X, Y)=0$. The transformation function $F(X, Y)$ in such methodologies is chosen from, and is estimated in, a class of sufficiently smooth functions that ensures the existence of the derivative $\bar{\beta}^{\prime}(1)$ and therefore correctness of the definition of scale elasticity $\varepsilon\left(X_{o}, Y_{o}\right)$ (Balk et al., 2015).

In nonparametric methodologies of efficiency and productivity analysis such as DEA, the implicit production frontier is not smooth and the derivative $\bar{\beta}^{\prime}(1)$ and the scale elasticity $\varepsilon\left(X_{o}, Y_{o}\right)$ are generally undefined. In such cases it is common to consider the notion of one-sided (left-hand and right hand) derivatives and scale elasticities. Provided the required one-sided derivatives exist (and conventionally allowing their infinite values corresponding to the possible "vertical" slopes of the facets of the production frontier), we have the following definition.

Definition 1. The one-sided scale elasticities at DMU $\left(X_{o}, Y_{o}\right)$ are defined as follows:

$$
\begin{aligned}
& \varepsilon^{+}\left(X_{o}, Y_{o}\right)=\bar{\beta}_{+}^{\prime}(1), \\
& \varepsilon^{-}\left(X_{o}, Y_{o}\right)=\bar{\beta}_{-}^{\prime}(1) .
\end{aligned}
$$

\footnotetext{
${ }^{3}$ We consider the function $\bar{\beta}(\alpha)$ undefined outside $\Gamma$. One may additionally define $\bar{\beta}(\alpha)=-\infty$ for all $\alpha \in \mathbb{R} \backslash \Gamma$, but such an extension is not used in the statements and proofs of the results in this paper.

${ }^{4}$ Let $\Gamma$ be the domain of $\bar{\beta}(\alpha)$, i.e., the set of all values $\alpha$ for which program (1) is feasible in $\beta$. Because technology $\mathcal{T}$ is assumed to be closed and have bounded output sets for each vector of inputs, the maximum $\bar{\beta}(\alpha)$ in (1) is attained and is finite, for all $\alpha \in \Gamma$. If technology $\mathcal{T}$ is convex, then $\Gamma$ is a closed interval (Podinovski, 2017).
} 
The one-sided scale elasticities exist for a very large spectrum of production technologies, including all polyhedral technologies of DEA (Podinovski et al., 2016) and, even more broadly, all convex technologies (Podinovski, 2017).

There exists extensive literature on the evaluation of one-sided scale elasticities $\varepsilon^{+}\left(X_{o}, Y_{o}\right)$ and $\varepsilon^{-}\left(X_{o}, Y_{o}\right)$ in different DEA technologies (Chambers \& Färe, 2008; Førsund \& Hjalmarsson, 2004; Fukuyama, 2000; Hadjicostas \& Soteriou, 2006; Sahoo \& Tone, 2015; Zelenyuk, 2013). Banker and Thrall (1992) show that, for the standard VRS technology, these scale elasticities can be evaluated based on the range of values of the dual variable corresponding to the convexity constraint in the envelopment model. A non-trivial generalization of this result to any polyhedral technology was recently obtained by Podinovski et al. (2016).

\subsection{Returns to scale}

The RTS characterization of DMUs located on the production frontier, i.e., satisfying Assumption 1, is based on the value of scale elasticity (2) or its one-sided analogues (3). In the former case, $\mathrm{DMU}_{o}$ is said to exhibit increasing, decreasing or constant RTS (IRS, DRS and CRS $)$ if $\varepsilon\left(X_{o}, Y_{o}\right)>1, \varepsilon\left(X_{o}, Y_{o}\right)<1$ and $\varepsilon\left(X_{o}, Y_{o}\right)=1$, respectively.

If the scale elasticity $\varepsilon\left(X_{o}, Y_{o}\right)$ is undefined, the RTS characterization may still be obtained based on the one-sided scale elasticities (3), provided they exist. In the case of VRS technology, Banker and Thrall (1992) define the RTS types as follows.

Definition 2. DMU $\left(X_{o}, Y_{o}\right)$ exhibits

(i) IRS if $1<\varepsilon^{+}\left(X_{o}, Y_{o}\right) \leq \varepsilon^{-}\left(X_{o}, Y_{o}\right)$;

(ii) DRS if $\varepsilon^{+}\left(X_{o}, Y_{o}\right) \leq \varepsilon^{-}\left(X_{o}, Y_{o}\right)<1$;

(iii) $\mathrm{CRS}$ if $\varepsilon^{+}\left(X_{o}, Y_{o}\right) \leq 1 \leq \varepsilon^{-}\left(X_{o}, Y_{o}\right)$.

The above definition of RTS also extends to all convex production technologies. As proved by Podinovski (2017), and similar to the case of the VRS technology, we always have

$$
\varepsilon^{+}\left(X_{o}, Y_{o}\right) \leq \varepsilon^{-}\left(X_{o}, Y_{o}\right)
$$

Therefore, the three types of RTS in Definition 2 exhaust all possibilities.

If technology $\mathcal{T}$ is not convex, the inequality (4) is not necessarily true and the three cases in Definition 2 may not describe all possibilities. For example, in a nonconvex technology, we may have

$$
\varepsilon^{+}\left(X_{o}, Y_{o}\right)>1>\varepsilon^{-}\left(X_{o}, Y_{o}\right),
$$

indicating that DMU $\left(X_{o}, Y_{o}\right)$ exhibits IRS if the input vector $X_{o}$ is proportionally increased and DRS if $X_{o}$ is proportionally reduced, which is impossible in the VRS and any other convex technology. ${ }^{5}$ We illustrate this possibility using a nonconvex technology in Example 3 below. Similar scenarios can also arise for piecewise linear approximations of $S$-shape production functions (Olesen and Ruggiero, 2014).

Remark 1. In the VRS technology, both one-sided scale elasticities (3) are always nonnegative. The same is true for any technology $\mathcal{T}$ that satisfies the assumption of free (strong) disposability of its inputs and outputs, or at least the assumption of joint weak disposability

\footnotetext{
${ }^{5}$ Podinovski (2004a, 2004b) explores these possibilities in the case of arbitrary technologies. This leads to the definition of a global RTS characterization indicative of the direction of resizing towards the most productive scale size in the sense on Banker (1984). The global RTS characterization is empirically investigated by Cesaroni, Kerstens, and Van de Woestyne (2017).
} 
of all inputs (Färe et al., 1983). In this case, the function $\bar{\beta}(\alpha)$ is nondecreasing and we always have $\varepsilon^{-}\left(X_{o}, Y_{o}\right) \geq \varepsilon^{+}\left(X_{o}, Y_{o}\right) \geq 0$. However, in the general case (including some convex technologies), the nonnegativity of one-sided scale elasticities cannot be assured as, for example, in technologies with weakly disposable good outputs (Mehdiloo and Podinovski, 2019). In such cases the DRS and CRS types of RTS may require a more refined definition accounting for the possibility of the negative scale elasticity. In our paper, we do not consider such possible extensions and focus on the standard Definition 2.

Remark 2. The scale elasticity $\varepsilon\left(X_{o}, Y_{o}\right)$ defined by (2), its one-sided analogues defined by (3) and the corresponding RTS types introduced by Definition 2 are (marginal) characteristics of production frontiers. By definition, these characteristics depend only on the model of technology and are independent of any efficiency measures that may be used with the given technology in the efficiency assessment of DMUs (such as input and output radial measures, hyperbolic measures or measures based on particular directional vectors). This is especially evident in the case of production technologies given by a known transformation function $F(X, Y)$. For such technologies, the scale elasticity is found by a straightforward formula involving partial derivatives of the transformation function - for a modern exposition, see, e.g., Balk et al. (2015, p. 100).

The independence of scale elasticity from any efficiency measures may be less obvious in nonparametric DEA models. For example, the evaluation of one-sided scale elasticities in the VRS technology is often based on the calculation of the maximal and minimal values of the dual variable to the convexity constraint of the envelopment program (Banker \& Thrall, 1992). These values depend on whether the model is solved in the input or output orientation. However, as shown by Førsund and Hjalmarsson $(2004)$, if $\operatorname{DMU}\left(X_{o}, Y_{o}\right)$ is efficient, and in line with the theoretical definition, the resulting one-sided scale elasticities do not depend on the orientation of the model and are the same in both cases. ${ }^{6}$

For inefficient DMUs, the methods based on solving DEA models with different efficiency measures evaluate the one-sided scale elasticities at the projection of DMU $\left(X_{o}, Y_{o}\right)$. Because such projections are usually different for different efficiency measures (e.g., the input and output radial projections of an inefficient DMU are generally different), the resulting onesided scale elasticities are also generally different.

\section{Embedded technologies}

Below we establish a relationship between the one-sided scale elasticities and RTS characterizations in two production technologies $\mathcal{T}$ and $\tilde{\mathcal{T}}$ specified in terms of the same sets of inputs and outputs, one of which is a subset of the other.

To see the relevance of this scenario to practical applications, assume that we have specified some technology $\mathcal{T}$ in terms of $m$ inputs and $s$ outputs, based on some set of observed DMUs. We may subsequently need to use a larger technology $\tilde{\mathcal{T}}$, for a number of reasons. First, we may decide to use a larger set of observed DMUs. Second, we may change some assumptions on which the model is based leading to a larger technology. For example, if technology $\mathcal{T}$ is based on the assumption of weak input disposability and $\tilde{\mathcal{T}}$ is strongly disposable, then the former technology is a subset of the latter. Finally, we may decide to incorporate additional information in the model leading to its expansion, for example, in the

\footnotetext{
${ }^{6}$ The assessment of scale elasticity based on the input-oriented VRS model is applicable only if DMU $\left(X_{o}, Y_{o}\right)$ is both input and output radial efficient, as otherwise the scale elasticity is evaluated at the input projection of this DMU. In contrast, the use of the output-oriented VRS model requires that DMU $\left(X_{o}, Y_{o}\right)$ be only output radial efficient, i.e., satisfy Assumption 1.
} 
form of weight restrictions $\mathcal{T}$ (see, e.g., Roll, Cook and Golany, 1991, and Podinovski, 2004c) or additional unobserved DMUs (Thanassoulis and Allen, 1998). Of course, the opposite modelling direction is also possible, i.e., starting with the larger technology $\tilde{\mathcal{T}}$, we may subsequently remove some observed DMUs or change the current production assumptions to the ones that result in a smaller technology $\mathcal{T}$.

To describe the above scenario formally, let $\mathcal{T} \subseteq \tilde{\mathcal{T}} \subset \mathbb{R}_{+}^{m} \times \mathbb{R}_{+}^{s}$ and let $\mathrm{DMU}_{o}$ be a member of both technologies. We first have to disallow the case in which $\mathrm{DMU}_{o}$ is output radial efficient, i.e., satisfies Assumption 1, only in the smaller technology $\mathcal{T}$ but is inefficient in the larger technology $\tilde{\mathcal{T}}$. In this case, the RTS characterization of $\mathrm{DMU}_{o}$ is correctly defined only in technology $\mathcal{T}$ and no further meaningful comparison between the two technologies is possible.

Let therefore $\mathrm{DMU}_{o}$ satisfy Assumption 1 in both technologies $\mathcal{T}$ and $\tilde{\mathcal{T}}$ (it is obviously sufficient to verify this only in the larger technology $\tilde{\mathcal{T}})$. Denote $\varepsilon^{-}\left(X_{o}, Y_{o}\right)$ and $\varepsilon^{+}\left(X_{o}, Y_{o}\right)$ the one-sided scale elasticities evaluated at $\mathrm{DMU}_{o}$ in $\mathcal{T}$. Similarly, denote $\tilde{\varepsilon}^{-}\left(X_{o}, Y_{o}\right)$ and $\tilde{\varepsilon}^{+}\left(X_{o}, Y_{o}\right)$ the one-sided scale elasticities evaluated in $\tilde{\mathcal{T}}$. Assuming that these one-sided scale elasticities exist (which, if we conventionally allow their infinite values, is always true in any convex technology), we have the following statement.

Theorem 1. Let DMU $\left(X_{o}, Y_{o}\right) \in \mathcal{T} \subseteq \tilde{\mathcal{T}}$, and let $\left(X_{o}, Y_{o}\right)$ satisfy Assumption 1 in technology $\tilde{\mathcal{T}}$ (and, therefore, in the smaller technology $\mathcal{T}$ ). Then

$$
\begin{aligned}
& \varepsilon^{+}\left(X_{o}, Y_{o}\right) \leq \tilde{\varepsilon}^{+}\left(X_{o}, Y_{o}\right), \\
& \varepsilon^{-}\left(X_{o}, Y_{o}\right) \geq \tilde{\varepsilon}^{-}\left(X_{o}, Y_{o}\right) .
\end{aligned}
$$

Podinovski and Førsund (2010, Proposition 8) establish inequalities (6) in the case when $\mathcal{T}$ is the conventional VRS technology expanded by the incorporation of additional observed DMUs. The above Theorem 1 extends this result to a much more general case.

The meaning of Theorem 1 should be intuitively clear. It can be explained using the conventional interpretations of scale elasticity and its one-sided analogues that follow from their definitions (2) and (3). Suppose, for example, that the right-hand scale elasticity $\varepsilon^{+}\left(X_{o}, Y_{o}\right)$ evaluated at DMU $\left(X_{o}, Y_{o}\right) \in \mathcal{T}$ is equal to 1.5. Suppose that we proportionally increase the input vector by $1 \%$, i.e., from $X_{o}$ to $1.01 X_{o}$, which for the sake of discussion is considered a marginally small increase. Then, if the resulting DMU operates efficiently, i.e., lies on the production frontier, its output vector is proportionally increased by $1.5 \%$, i.e., from $Y_{o}$ to $1.015 Y_{o}$. Because technology $\mathcal{T}$ is a subset of $\tilde{\mathcal{T}}$, the increase of the output vector $Y_{o}$ in the latter technology would be no less than in technology $\mathcal{T}$. Therefore, for the right-hand scale elasticity in technology $\tilde{\mathcal{T}}$ we have $\tilde{\varepsilon}^{+}\left(X_{o}, Y_{o}\right) \geq 1.5$, which explains the first inequality in (6).

Similarly, consider the left-hand scale elasticity evaluated at DMU $\left(X_{o}, Y_{o}\right)$ and suppose that $\varepsilon^{-}\left(X_{o}, Y_{o}\right)=2.5$. This means that a proportional reduction of the input vector by $1 \%$, i.e., from $X_{o}$ to $0.99 X_{o}$ would cause the output vector to be reduced by $2.5 \%$, from $Y_{o}$ to $0.975 Y_{o}$, provided the resulting DMU remains on the production frontier of technology $\mathcal{T}$. In the larger technology $\tilde{\mathcal{T}}$ the reduction of the output vector cannot be more than by $2.5 \%$, because every output vector producible from the input vector $0.99 X_{o}$ in technology $\mathcal{T}$ is also producible in the larger technology $\tilde{\mathcal{T}}$. Therefore, we have $\tilde{\varepsilon}^{-}\left(X_{o}, Y_{o}\right) \leq 2.5$, which explains the second inequality in (6).

The statement of Theorem 1 and its interpretation are further illustrated by Examples 13 considered below.

Now consider the case of convex technologies. (Formally, it suffices to assume that only technology $\tilde{\mathcal{T}}$ is convex.) In this case, restating inequality (4) for the one-sided scale 
elasticities $\tilde{\varepsilon}^{-}\left(X_{o}, Y_{o}\right)$ and $\tilde{\varepsilon}^{+}\left(X_{o}, Y_{o}\right)$, and taking into account $(6)$, we have

$$
\varepsilon^{+}\left(X_{o}, Y_{o}\right) \leq \tilde{\varepsilon}^{+}\left(X_{o}, Y_{o}\right) \leq \tilde{\varepsilon}^{-}\left(X_{o}, Y_{o}\right) \leq \varepsilon^{-}\left(X_{o}, Y_{o}\right) .
$$

This last inequality implies that we have the following embedding of the two intervals for scale elasticity in the two technologies:

$$
\left[\tilde{\varepsilon}^{+}\left(X_{o}, Y_{o}\right), \tilde{\varepsilon}^{-}\left(X_{o}, Y_{o}\right)\right] \subseteq\left[\varepsilon^{+}\left(X_{o}, Y_{o}\right), \varepsilon^{-}\left(X_{o}, Y_{o}\right)\right]
$$

The established relationships lead to the following implications regarding the RTS characterization of $\mathrm{DMU}_{o}$ in the two technologies.

We first consider the case in which the RTS characterization of $\mathrm{DMU}_{o}$ in the smaller technology $\mathcal{T}$ is known and ask, what does this tell us about its RTS type in the larger technology $\tilde{\mathcal{T}}$ ?

Corollary 1. Let technology $\tilde{\mathcal{T}}$ be convex and let $D M U\left(X_{o}, Y_{o}\right) \in \mathcal{T} \subseteq \tilde{\mathcal{T}}$ satisfy Assumption 1 in technology $\tilde{\mathcal{T}}$. Then

(i) If $D M U_{o}$ exhibits IRS in technology $\mathcal{T}$, then it exhibits IRS in the larger technology $\tilde{\mathcal{T}}$.

(ii) If DMU $U_{o}$ exhibits DRS in technology $\mathcal{T}$, then it exhibits DRS in the larger technology $\tilde{\mathcal{T}}$.

Remark 3. If $\mathrm{DMU}_{o}$ exhibits CRS in technology $\mathcal{T}$, it is generally not possible to say what type of RTS it exhibits in the larger technology $\tilde{\mathcal{T}}$, as all three types are possible. We illustrate these possibilities in the examples below. ${ }^{7}$

We now assume that we have obtained the RTS type of $\mathrm{DMU}_{o}$ in the larger technology $\tilde{\mathcal{T}}$ and are interested in its RTS type in the smaller technology $\tilde{\mathcal{T}}$.

Corollary 2. Let technology $\tilde{\mathcal{T}}$ be convex and let $D M U\left(X_{o}, Y_{o}\right) \in \mathcal{T} \subseteq \tilde{\mathcal{T}}$ satisfy Assumption 1 in technology $\tilde{\mathcal{T}}$. Then

(i) If DMU exhibits CRS in technology $\tilde{\mathcal{T}}$, then it exhibits CRS in the smaller technology $\mathcal{T}$.

(ii) If DMU $U_{o}$ exhibits IRS in technology $\tilde{\mathcal{T}}$, then it exhibits either IRS or CRS in the smaller technology $\mathcal{T}$ but cannot exhibit DRS.

(iii) If $D M U_{o}$ exhibits DRS in technology $\tilde{\mathcal{T}}$, then it exhibits either DRS or CRS in the smaller technology $\mathcal{T}$ but cannot exhibit IRS.

Let us note that, if the production frontier is smooth, the scale elasticity $\varepsilon\left(X_{o}, Y_{o}\right)$ defined by (2) may be regarded as a measure of strength of the RTS type of $\mathrm{DMU}_{o}$. For example, suppose that the scale elasticity evaluated at some DMUs $A$ and $B$ is equal to 1.1 and 2.5, respectively. Then both DMUs exhibit IRS but the latter DMU exhibits a much stronger response of its output vector to marginal proportional changes of its input vector than the former, whose classification is not very different from CRS.

If the production frontier is not smooth, the strength of the RTS type of $\mathrm{DMU}_{o}$ is represented by the interval $\left[\varepsilon^{+}\left(X_{o}, Y_{o}\right), \varepsilon^{-}\left(X_{o}, Y_{o}\right)\right]$. If technology $\mathcal{T}$ is convex, this interval

\footnotetext{
${ }^{7}$ As noted in Section 2, the three types of IRS, DRS and CRS of Definition 2 provide a complete characterization of RTS provided the inequality (4) is satisfied, which is always true in a convex technology. It is interesting to note that, under the conditions of Corollaries 1 or 2, Definition 2 describes all possibilities even if $\mathcal{T}$ is not convex. Indeed, because technology $\tilde{\mathcal{T}}$ is convex, as shown, we have inequality (7) which in turn implies (4). Therefore, Definition 2 gives a complete characterization of RTS at DMU $\left(X_{o}, Y_{o}\right)$.
} 
Table 1: DMUs in Example 1.

\begin{tabular}{ccc}
\hline DMU & Input $X$ & Output $Y$ \\
\hline$A$ & 3 & 2 \\
$B$ & 6 & 6 \\
$C$ & 12 & 8 \\
$D$ & 4 & 4.5 \\
$F$ & 9 & 7.5 \\
\hline
\end{tabular}

Table 2: One-sided scale elasticities in Example 1.

\begin{tabular}{lcccc}
\hline & \multicolumn{2}{c}{ Technology } & $\mathcal{T}_{\text {VRS }}$ & \multicolumn{2}{c}{ Technology } & $\tilde{\mathcal{T}}_{\text {VRS }}$ \\
\cline { 2 - 5 } DMU & $\varepsilon^{+}$ & $\varepsilon^{-}$ & $\tilde{\varepsilon}^{+}$ & $\tilde{\varepsilon}^{-}$ \\
\hline$A$ & 2 & $+\infty$ & 3.75 & $+\infty$ \\
$B$ & 0.33 & 1.33 & 0.5 & 0.75 \\
$C$ & 0 & 0.5 & 0 & 0.25 \\
\hline
\end{tabular}

coincides with the range of scale elasticities measured along different supporting hyperplanes to $\mathcal{T}$.

Taking into account the embedding (8), it is now clear that, even if the type of RTS of $\mathrm{DMU}_{o}$ in technology $\tilde{\mathcal{T}}$ is the same as in $\mathcal{T}$ (as, for example, in the cases (i) and (ii) of Corollary 1 ), the strength of this characterization is generally more precisely defined (by a narrower range between the one-sided scale elasticities) in the larger technology $\tilde{\mathcal{T}}$ than in technology $\mathcal{T}$.

Remark 4. Theorem 1 becomes more precise in the case of smooth production frontiers for which the one-sided scale elasticities are equal to each other and coincide with the standard scale elasticity $\varepsilon\left(X_{o}, Y_{o}\right)$ in technology $\mathcal{T}$ and $\tilde{\varepsilon}\left(X_{o}, Y_{o}\right)$ in technology $\tilde{\mathcal{T}}$. Indeed, in this case, under the assumptions of Theorem 1 , we simply have:

$$
\varepsilon\left(X_{o}, Y_{o}\right)=\tilde{\varepsilon}\left(X_{o}, Y_{o}\right)
$$

This equality means that the type of RTS exhibited by $\mathrm{DMU}_{o}$ in technology $\mathcal{T}$ does not change and remains the same in the larger technology $\tilde{\mathcal{T}}$ (provided this $\mathrm{DMU}_{o}$ is output radial efficient in both technologies).

The following three examples illustrate the results obtained in this section in which the expanded technology $\tilde{\mathcal{T}}$ is obtained from technology $\mathcal{T}$ in three different ways: by adding additional observed DMUs, by incorporating additional information about the production process in the form of weight restrictions (trade-offs) and by changing the underlying set of production assumptions.

Example 1. Consider the VRS technology $\mathcal{T}_{\text {VRS }}$ with a single input and a single output generated by three observed DMUs $£ A, B$ and $C$ shown in Table 1 . Technology $\mathcal{T}_{\text {VRS }}$ is shown as the shaded area in Figure 1. 


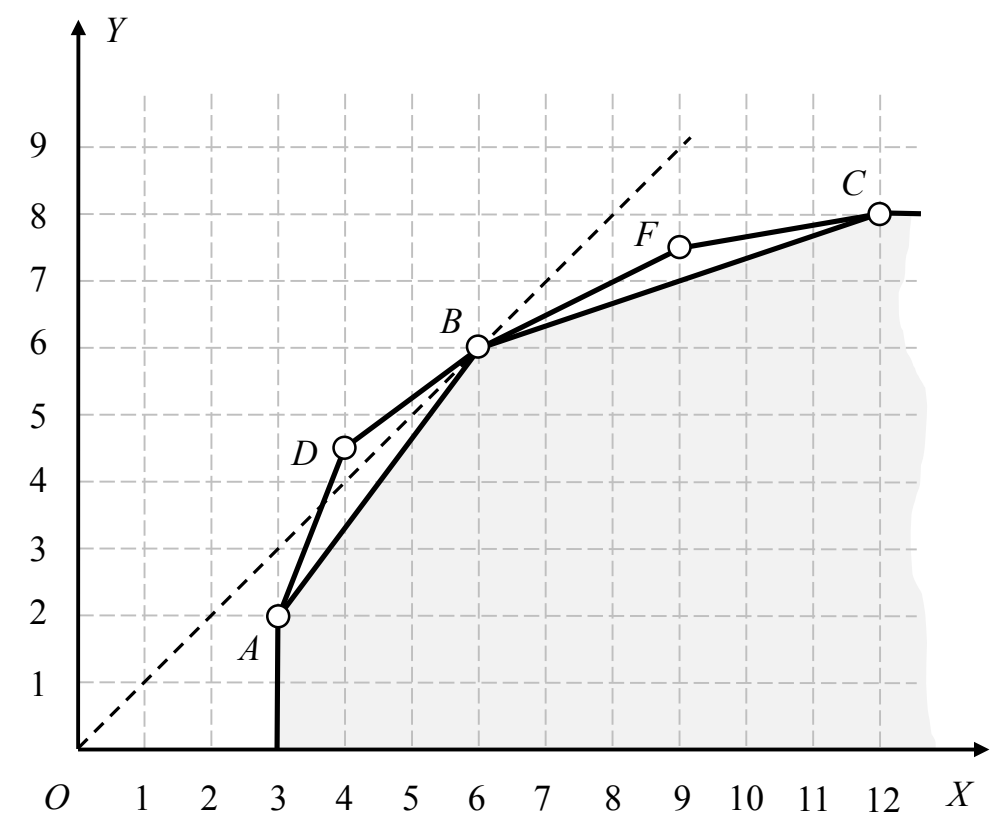

Figure 1: Technologies $\mathcal{T}_{\text {VRS }}$ and $\tilde{\mathcal{T}}_{\text {VRS }}$ in Example 1.

Suppose that we have added two further DMUs $D$ and $F$ to the data set, as shown in Table 1. The VRS technology $\tilde{\mathcal{T}}_{\text {VRS }}$ generated by all five observe DMUs is the area in the nonnegative orthant $\mathbb{R}_{+}^{2}$ below and to the right of the broken line $A D B F C$ in Figure 1 , which includes technology $\mathcal{T}_{\text {VRS }}$ as a subset.

DMUs $A, B$ and $C$ are output radial efficient, i.e., satisfy Assumption 1, in both technologies $\mathcal{T}_{\text {VRS }}$ and $\tilde{\mathcal{T}}_{\text {VRS }}$. Table 2 shows the one-sided scale elasticities evaluated at these DMUs in the two technologies (the values are rounded). ${ }^{8}$ Note that these values are consistent with the inequalities (6) stated in Theorem 1 and the further results given by formulae (7) and (8).

Note that, in technology $\mathcal{T}_{\text {VRS }}$, DMU $A$ exhibits IRS and DMU $C$ exhibits DRS. In line with statements (i) and (ii) of Corollary 1, these two DMUs keep their RTS type in the larger technology $\tilde{\mathcal{T}}_{\text {VRS }}$. However, DMU $B$ exhibits CRS in technology $\mathcal{T}_{\text {VRS }}$ and DRS in technology $\tilde{\mathcal{T}}_{\text {VRS }}$, which illustrates Remark 3.

Example 2. The shaded area in Figure 2 shows the VRS technology $\mathcal{T}_{\text {VRS }}$ generated by two DMUs $K=(3,4)$ and $L=(5,5)$, where the first component is input $X$ and the second is output $Y$. To be specific, suppose that we decided to assess the output radial efficiency of these DMUs using the multiplier VRS model based on this technology. Let $v_{1}$ and $u_{1}$ be, respectively, the input and output weights used in this model (together with the signfree variable corresponding to the convexity constraint of the envelopment program). In this model, both DMUs $K$ and $L$ are output radial efficient and, therefore, satisfy Assumption 1.

In order to improve the discrimination of the VRS model, suppose that we have decided to incorporate the following two weight restrictions:

$$
v_{1} \leq 4 u_{1}, \quad v_{1} \geq 2 u_{1} .
$$

\footnotetext{
${ }^{8}$ The values shown in Table 2 can be verified by the known formula according to which the scale elasticity evaluated at $\mathrm{DMU}_{o}$ is equal to the ratio of its marginal to average productivity. For example, the right-hand marginal productivity at DMU $A$ in technology $\mathcal{T}_{\text {VRS }}$ is the slope of the line $A B$ equal to $4 / 3$. The average productivity of $A$ is $2 / 3$. Therefore, the right-hand scale elasticity at DMU $A$ is equal to $(4 / 3) /(2 / 3)=2$.
} 


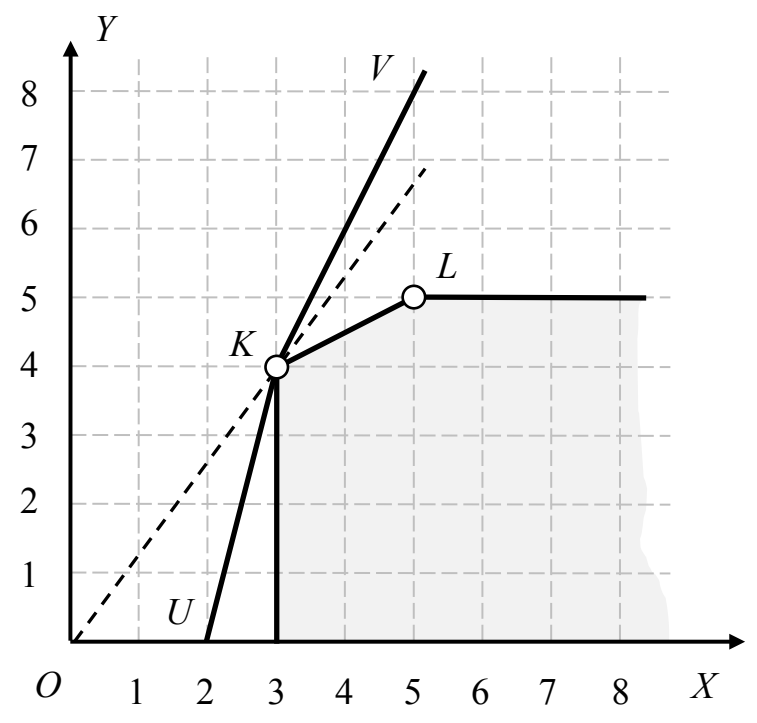

Figure 2: Technologies $\mathcal{T}_{\text {VRS }}$ and $\tilde{\mathcal{T}}_{\text {VRS }}$ in Example 2.

As shown by Podinovski (2004c), the incorporation of weight restrictions (9) is equivalent to the specification of trade-offs between the input and output leading to the expansion of technology. Namely, the first weight restriction in (9) is equivalent to the specification of the following trade-off: it is possible to reduce the input by one unit provided the output is reduced by four units. This information expands technology $\mathcal{T}_{\text {VRS }}$ by adding the line $U K$ and the area below it. Similarly, the second weight restriction in (9) is equivalent to the specification of the following trade-off: it is possible to increase the output by two units, provided the input is increased by one unit. This judgement adds the line $K V$, and the area below it, to the technology. The resulting technology $\tilde{\mathcal{T}}_{\text {VRS }}$ is the area below and to the right of the broken line $U K V$, which is its efficient frontier.

Note that DMU $L$ is no longer output radial efficient in the expanded technology $\tilde{\mathcal{T}}_{\text {VRS }}$ and is not characterized in terms of RTS. DMU $K$ exhibits CRS in the original technology $\mathcal{T}_{\text {VRS }}$, which becomes IRS in the expanded technology $\tilde{\mathcal{T}}_{\text {VRS }}$. This finding illustrates Remark 3.

Example 3. This example illustrates our results in the case of a nonconvex technology. Assume that there are three observed DMUs $P, Q$ and $R$ evaluated on a single input and a single output. Figure 3 shows three different technologies discussed in this example.

Technology $\mathcal{T}_{\text {FDH }}$ is the free disposal hull (FDH) technology of Deprins, Simar and Tulkens (1984) generated by the three observed DMUs. It coincides with the area below and to the right of the broken punctured line $L P N Q M R Z$.

Technology $\mathcal{T}_{\text {FDH-TO }}$ is obtained from $\mathcal{T}_{\text {FDH }}$ by the incorporation of the following two production trade-offs. Trade-off 1 states that it is possible to increase the output by 0.25 provided the input is increased by 1 . This trade-off generates the lines $P U, Q V$ and $R W$. Trade-off 2 states that it is possible to reduce the input by 0.25 provided the output is reduced by 1 . This trade-off generates the lines $P S, Q U$ and $R V$. For example, DMU $S$ is obtained from DMU $P$ by the application of trade-off 2 twice, i.e., by reducing the input of DMU $P$ by 0.5 and the output by 2 . This results in the "inactive" DMU $S$ that produces no output. Technology $\mathcal{T}_{\text {FDH-TO }}$ is shown as the shaded area below and to the right of the solid broken line $S P U Q V R W$ which is its strongly efficient frontier.

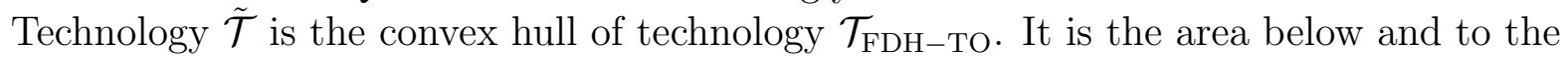
right of the broken line $S P Q R W$ which is its strongly efficient frontier. This technology 


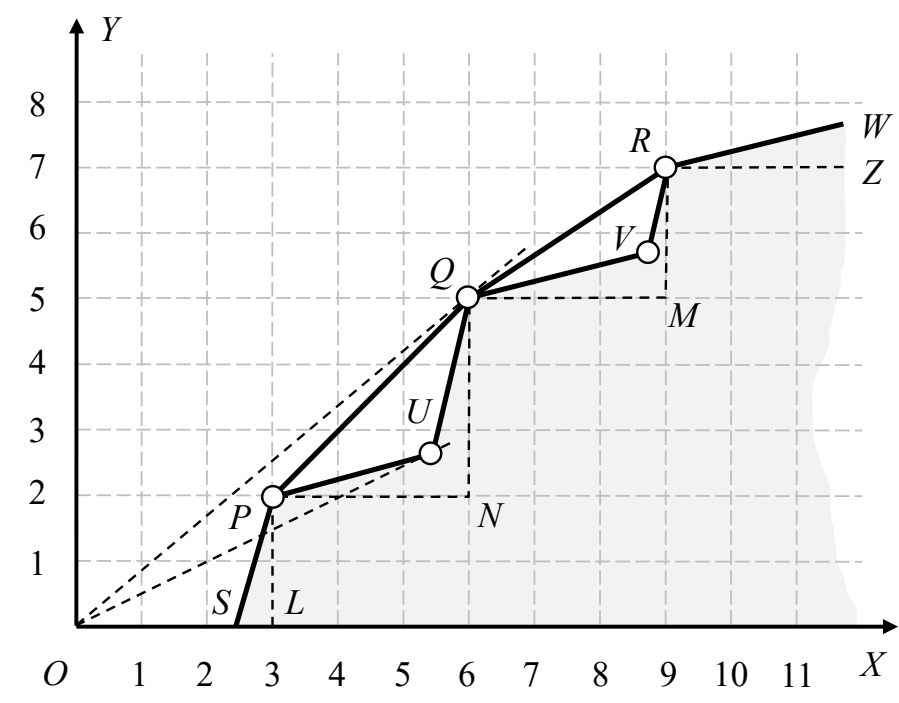

Figure 3: Technologies $\mathcal{T}_{\text {FDH }}, \mathcal{T}_{\text {FDH-TO }}$ and $\tilde{\mathcal{T}}$ in Example 3.

can alternatively be defined as the VRS technology generated by the observed DMUs $P, Q$ and $R$ (represented by the area below and to the right of the broken line LPQRZ) which has subsequently been expanded by the two trade-offs specified above. These trade-offs are equivalent to the specification of the two weight restrictions $0.25 u_{1} \leq v_{1}$ and $u_{1} \geq 0.25 v_{1}$ incorporated in the multiplier VRS models, where $u_{1}$ and $v_{1}$ are the output and input weights, respectively (Podinovski, 2004c).

Note that all three observed DMUs $P, Q$ and $R$ are output radial efficient in all three of the above technologies, i.e., they satisfy Assumption 1 in all of them. According to Definition 2, in technology $\mathcal{T}_{\text {FDH-TO }}$, all three observed DMUs exhibit CRS. ${ }^{9}$

We can also formally apply Definition 2 to the observed DMUs in technology $\mathcal{T}_{\text {FDH }}$. In this case, the left-hand scale elasticity at each DMU $P, Q$ and $R$ is equal to $+\infty$ (which corresponds to the vertical lines $P L, Q N$ and $R M$ ) and the right-hand scale elasticity is equal to zero. According to Definition 2, all these DMUs also exhibit CRS. ${ }^{10}$

Now consider technology $\tilde{\mathcal{T}}$. Because the latter technology is convex, the assumption of Corollaries 1 and 2, in which we can take $\mathcal{T}=\mathcal{T}_{\text {FDH }}$ or $\mathcal{T}=\mathcal{T}_{\text {FDH-TO }}$, are satisfied. As an illustration to Remark 3, note that, in technology $\tilde{\mathcal{T}}$, DMU $P$ exhibits IRS, $Q$ exhibits CRS and $R$ exhibits DRS.

Finally, as an illustration to our discussion of the incompleteness of Definition 2 of RTS in nonconvex technologies, consider DMUs $U$ and $V$. Both are strongly efficient and therefore satisfy Assumption 1 in the nonconvex technology $\mathcal{T}_{\text {FDH-TO }}$. It is straightforward to show that the one-sided scale elasticities evaluated at both DMUs satisfy inequality (5). Therefore, these two DMUs exhibit IRS if their inputs are increased and DRS if their inputs are reduced, which is impossible in a convex technology.

\footnotetext{
${ }^{9}$ For example, as highlighted in Footnote 8, the left-hand scale elasticity at DMU $P$ is equal to the ratio of its left-hand marginal productivity (the slope of the line $S P$ ) to its average productivity, i.e., $4 /(2 / 3)=6>1$. Similarly, the right-hand scale elasticity at $P$ is equal to $0.25 /(2 / 3)=0.375<1$. By Definition 2, DMU $P$ exhibits CRS.

${ }^{10}$ Cesaroni et al. (2017) point out that the fact that all efficient DMUs in the FDH technology exhibit CRS is a consequence of the discontinuity of the function of ray average productivity. They develop an alternative definition of RTS aimed at obtaining a more informative characterization of RTS in FDH.
} 
In line with an analogous situation arising in the global RTS characterization introduced by Podinovski (2004a, 2004b) and generalizing the extended local RTS characterization of smooth production frontiers suggested by Cesaroni et al. (2017), we can regard DMUs $U$ and $V$ as exhibiting the sub-constant type of local RTS (S-CRS). As noted, this type cannot occur in convex production technologies.

\section{Technologies with aggregated and omitted inputs and outputs}

\subsection{Motivation and definitions}

In this section we consider the scenario in which technology $\hat{\mathcal{T}}$ is obtained from technology $\mathcal{T}$ by a linear aggregation of some of its inputs or outputs. For example, several inputs of technology $\mathcal{T}$ may represent different costs that are added together and counted as one input in technology $\hat{\mathcal{T}}$. Another typical example is the aggregation of several outputs representing different products to a single output representing the overall monetary value of all products. This aggregation is typically performed with different coefficients attached to different products that represent their unit prices.

A special case of the described aggregation is the scenario in which some inputs or outputs used in technology $\mathcal{T}$ are omitted in technology $\hat{\mathcal{T}}$. Formally, this corresponds to the aggregation of the omitted inputs or outputs with one of the remaining inputs or, respectively, outputs, where the weights of the omitted measures are taken equal to zero and the weight of the remaining measure is equal to one.

Let technology $\mathcal{T}$ have $m \geq 1$ inputs and $s \geq 1$ outputs, and let the input and output vectors be stated as follows:

$$
\begin{aligned}
& X=\left(x_{1}, x_{2}, \ldots, x_{m^{\prime}-1}, x_{m^{\prime}}, \ldots, x_{m}\right)^{\top}, \\
& Y=\left(y_{1}, y_{2}, \ldots, y_{s^{\prime}-1}, y_{s^{\prime}}, \ldots, y_{s}\right)^{\top},
\end{aligned}
$$

where $1 \leq m^{\prime} \leq m$ and $1 \leq s^{\prime} \leq s$.

Consider the linear aggregation of inputs $i=m^{\prime}, \ldots, m$, and outputs $r=s^{\prime}, \ldots, s$, taken with the weights $a_{i} \geq 0$ and $b_{r} \geq 0$, respectively, and assume that at least one of the weights $a_{i}$ and at least one the weights $b_{r}$ are strictly positive. This transforms DMU $(X, Y)$ in $(10)$ to the DMU $(\hat{X}, \hat{Y})$ with $m^{\prime}$ inputs and $s^{\prime}$ outputs as follows:

$$
\begin{aligned}
& \hat{X}=\left(x_{1}, x_{2}, \ldots, x_{m^{\prime}-1}, \sum_{i=m^{\prime}}^{m} a_{i} x_{i}\right)^{\top}, \\
& \hat{Y}=\left(y_{1}, y_{2}, \ldots, y_{s^{\prime}-1}, \sum_{r=s^{\prime}}^{s} b_{r} y_{r}\right)^{\top} .
\end{aligned}
$$

Note that we allow $m^{\prime}=1$ and $s^{\prime}=1$. In such cases all inputs and, respectively, all outputs are aggregated, and the components $x_{1}, x_{2}, \ldots, x_{m^{\prime}-1}$ and, respectively, $y_{1}, y_{2}, \ldots$, $y_{s^{\prime}-1}$ are omitted from (10) and (11).

Let technology $\hat{\mathcal{T}} \subset \mathbb{R}_{+}^{m^{\prime}} \times \mathbb{R}_{+}^{s^{\prime}}$ be obtained from technology $\mathcal{T} \subset \mathbb{R}_{+}^{m} \times \mathbb{R}_{+}^{s}$ by the described aggregation of the inputs and outputs of the latter, i.e.,

$$
\hat{\mathcal{T}}=\{(\hat{X}, \hat{Y}) \mid \exists(X, Y) \in \mathcal{T}:(\hat{X}, \hat{Y}) \text { obtained by }(11) \text { from }(X, Y)\} .
$$

Below, we refer to technology $\hat{\mathcal{T}}$ as the aggregated technology. ${ }^{11}$

\footnotetext{
${ }^{11}$ It is clear that the aggregation (11) needs to be meaningful and that the resulting technology $\hat{\mathcal{T}}$ should
} 


\subsection{Construction of aggregated technologies}

The theoretical definition (12) of the aggregated technology $\hat{\mathcal{T}}$ is not useful for practical applications for which a more operational statement is needed. In this section we show that, for the VRS and some other conventional technologies, the operational statements of their aggregated variants are straightforward.

Let $\mathcal{T}_{\text {VRS }}$ be the VRS technology of Banker et al. (1984) generated by the finite set of observed DMUs $\left(X_{j}, Y_{j}\right) \in \mathbb{R}_{+}^{m} \times \mathbb{R}_{+}^{s}, j=1, \ldots, n$. Let DMUs $\left(\hat{X}_{j}, \hat{Y}_{j}\right) \in \mathbb{R}_{+}^{m^{\prime}} \times \mathbb{R}_{+}^{s^{\prime}}$ be obtained by the aggregation of the observed DMUs $\left(X_{j}, Y_{j}\right)$ using formula (11).

Note that now we have two potentially different technologies. The first is technology $\hat{\mathcal{T}}_{\text {VRS }}$ theoretically defined by formula (12) and whose explicit operational statement is unknown. The second is the conventional VRS technology generated in the space $\mathbb{R}_{+}^{m^{\prime}} \times \mathbb{R}_{+}^{s^{\prime}}$ by the aggregated observed DMUs $\left(\hat{X}_{j}, \hat{Y}_{j}\right), j=1, \ldots, n$ whose statement is straightforward. The next result is not trivial and shows that these two technologies are the same.

Proposition 1. The aggregated technology $\hat{\mathcal{T}}_{\text {VRS }}$ is the conventional VRS technology generated by the set of aggregated observed DMUs $\left(\hat{X}_{j}, \hat{Y}_{j}\right), j=1, \ldots, n$.

It is easy to show that a similar statement to that of Proposition 1 remains true for the constant, nonincreasing and nondecreasing returns-to-scale technologies (Charnes, Cooper, \& Rhodes, 1978; Färe et al., 1983), and also for FDH (Deprins et al., 1984). Namely their aggregated variants obtained by formula (12) coincide with the corresponding standard technologies generated by the sets of aggregated observed DMUs $\left(\hat{X}_{j}, \hat{Y}_{j}\right) \in \mathbb{R}_{+}^{m^{\prime}} \times \mathbb{R}_{+}^{s^{\prime}}$, $j=1, \ldots, n$. This means that, in practice, construction of the aggregated analogues of any of above technologies is straightforward and requires only the aggregation of the observed DMUs. We illustrate the use of Proposition 1 in the case of a VRS technology in Example 4.

In a more general case, an analogue of Proposition 1 may not be true. For example, for the VRS technologies expanded by the specification of weight restrictions (or equivalent to them dual production trade-offs), a direct analogue of Proposition 1 and its proof remain true if the weight restrictions are specified only for the inputs and outputs that are not aggregated (or omitted). However, in the general case of weight restrictions, although the required aggregated technology is theoretically defined by (12), its operational statement is unclear. Exploring this issue, as well as the construction of the aggregated analogues of many other known polyhedral DEA technologies, is a complex task which lies outside the scope of this paper.

\subsection{Aggregation and efficiency}

We now revert to the case of general technology $\mathcal{T}$ and its aggregated variant $\hat{\mathcal{T}}$. Before considering the relationship between the RTS characterizations in these technologies, let us first show that the use of the latter technology in efficiency assessment leads to a generally better discrimination on efficiency than the use of the former technology.

Consider any $\mathrm{DMU}_{o}=\left(X_{o}, Y_{o}\right)$ in technology $\mathcal{T}$. Let $\widehat{\mathrm{DMU}}_{o}=\left(\hat{X}_{o}, \hat{Y}_{o}\right)$ be obtained from $\mathrm{DMU}_{o}$ by the aggregation formula (11) and, therefore, be a member of technology $\hat{\mathcal{T}}$. To be

satisfy the same minimal set of properties as the original technology $\mathcal{T}$, i.e., it should be a closed set and have bounded output sets for each input vector. The former property is always true, provided $\mathcal{T}$ is closed, but the latter needs verifying. For example, let $\mathcal{T}$ be described parametrically as the set of all triplets $(1, \alpha, \alpha)$, where $\alpha \geq 0$, the first two components are inputs and the third component is output. If we simply exclude the second input, the resulting technology $\hat{\mathcal{T}}$ would be described as the set of all input-output combinations $(1, \alpha)$ and have an unbounded output set for the input equal to one. 
specific, consider the case of output radial efficiency. The efficiency of $\mathrm{DMU}_{o}$ in this case is the inverse of the optimal value $\beta^{*}$ of the following program: ${ }^{12}$

$$
\beta^{*}=\max \left\{\beta \mid\left(X_{o}, \beta Y_{o}\right) \in \mathcal{T}, \beta \in \mathbb{R}\right\}
$$

Similarly, the output radial efficiency of $\widehat{\mathrm{DMU}}_{o}$ in technology $\hat{\mathcal{T}}$ is obtained as the inverse of the optimal value $\hat{\beta}^{*}$ in the program

$$
\hat{\beta}^{*}=\max \left\{\beta \mid\left(\hat{X}_{o}, \beta \hat{Y}_{o}\right) \in \hat{\mathcal{T}}, \beta \in \mathbb{R}\right\} .
$$

Proposition 2. $\hat{\beta}^{*} \geq \beta^{*}$.

Proposition 2 states that the output radial efficiency of $\widehat{\mathrm{DMU}}_{o}$ in technology $\hat{\mathcal{T}}$ (the inverse of the optimal $\hat{\beta}^{*}$ ) is not higher than the efficiency of $\mathrm{DMU}_{o}$ in technology $\mathcal{T}$. To summarize this result in words, the use of the aggregated technology $\hat{\mathcal{T}}$ leads to a generally better discrimination on efficiency than the use of technology $\mathcal{T}$. A special case of this result is the well-known fact that the removal of some inputs or outputs in the standard VRS and CRS DEA models generally improves the discrimination of the model.

A similar result for the input radial efficiency is also known in the literature. Färe et al. (2004) establish an input-oriented analogue of Proposition 2 for the standard VRS and CRS technologies, using a more specific framework of cost functions. They also obtain an upper bound on the difference between the input radial efficiencies evaluated in the original (disaggregated) and the aggregated technologies, which is referred to as the aggregation bias. These results are further generalized by Zelenyuk (2019).

Of immediate importance to us is the following implication of Proposition 2.

Corollary 3. If $\widehat{\mathrm{DMU}}_{\text {o }}$ satisfies Assumption 1, i.e., is output radial efficient in technology $\hat{\mathcal{T}}$, then $D M U_{o}$ satisfies Assumption 1 in technology $\mathcal{T}^{13}$

\subsection{Aggregation and RTS}

Let $\varepsilon^{-}\left(X_{o}, Y_{o}\right)$ and $\varepsilon^{+}\left(X_{o}, Y_{o}\right)$ be the one-sided scale elasticities evaluated at $\mathrm{DMU}_{o}$ in technology $\mathcal{T}$. Similarly, let $\hat{\varepsilon}^{-}\left(\hat{X}_{o}, \hat{Y}_{o}\right)$ and $\hat{\varepsilon}^{+}\left(\hat{X}_{o}, \hat{Y}_{o}\right)$ be the one-sided scale elasticities evaluated at $\widehat{\mathrm{DMU}}_{o}$ in technology $\hat{\mathcal{T}}$. Assuming that these one-sided scale elasticities exist (which is always true in convex technologies), ${ }^{14}$ we have the following statement.

Theorem 2. Let $\operatorname{DMU}\left(\hat{X}_{o}, \hat{Y}_{o}\right) \in \hat{\mathcal{T}}$ satisfy Assumption 1 in technology $\hat{\mathcal{T}}$ (in which case, by Corollary 3, DMU $\left(X_{o}, Y_{o}\right)$ satisfies Assumption 1 in technology $\left.\mathcal{T}\right)$. Then

$$
\begin{aligned}
& \varepsilon^{+}\left(X_{o}, Y_{o}\right) \leq \hat{\varepsilon}^{+}\left(\hat{X}_{o}, \hat{Y}_{o}\right), \\
& \varepsilon^{-}\left(X_{o}, Y_{o}\right) \geq \hat{\varepsilon}^{-}\left(\hat{X}_{o}, \hat{Y}_{o}\right) .
\end{aligned}
$$

\footnotetext{
${ }^{12}$ This program is program (1) stated with $\alpha=1$ and whose optimal value is renamed as $\beta^{*}$, i.e., we have $\beta^{*}=\bar{\beta}(1)$.

${ }^{13}$ The converse is generally not true. For example, consider the VRS technology $\mathcal{T}$ generated by DMUs $A=(1,2,2)$ and $B=(1,5,1)$, where the first component is input and the last two components are outputs. Both DMUs are output radial efficient, i.e., satisfy Assumption 1. Let $\hat{\mathcal{T}}$ be obtained from $\mathcal{T}$ by adding the two outputs together. The corresponding DMUs are $\hat{A}=(1,4)$ and $\hat{B}=(1,6)$, and the former is not output radial efficient in $\hat{\mathcal{T}}$. A similar illustration is obtained if the second output is simply removed from the model.

${ }^{14}$ It is easy to prove that, if technology $\mathcal{T}$ is convex, then technology $\hat{\mathcal{T}}$ is also convex, but the converse is not necessarily true.
} 
Theorem 2 allows an intuitive explanation. To be specific, let the right-hand scale elasticity $\varepsilon^{+}\left(X_{o}, Y_{o}\right)$ evaluated at $\operatorname{DMU}\left(X_{o}, Y_{o}\right) \in \mathcal{T}$ be equal to 1.5. Consider proportional increase of the input vector by $1 \%$, i.e., from $X_{o}$ to $1.01 X_{o}$. For the sake of discussion, let us view this increase as marginally small. Then the maximal increase of the output vector possible in technology $\mathcal{T}$ is $1.5 \%$, which corresponds to the resulting DMU $\left(1.01 X_{o}, 1.015 Y_{o}\right)$ being output radial efficient in $\mathcal{T}$. Because both DMUs $\left(X_{o}, Y_{o}\right)$ and $\left(1.01 X_{o}, 1.015 Y_{o}\right)$ are in technology $\mathcal{T}$, their aggregated variants $\left(\hat{X}_{o}, \hat{Y}_{o}\right)$ and $\left(1.01 \hat{X}_{o}, 1.015 \hat{Y}_{o}\right)$ obtained by formula (11) are by definition (12) in technology $\hat{\mathcal{T}}$. We no longer know if the latter DMU $\left(1.01 \hat{X}_{o}, 1.015 \hat{Y}_{o}\right)$ is efficient in $\hat{\mathcal{T}}$. However, the fact that $\left(1.01 \hat{X}_{o}, 1.015 \hat{Y}_{o}\right) \in \hat{\mathcal{T}}$ means that a proportional increase of the aggregated input vector $\hat{X}_{o}$ by $1 \%$ is sufficient for the increase of the production of the aggregated output vector $\hat{Y}_{o}$ by at least $1.5 \%$. This implies that $\hat{\varepsilon}^{+}\left(\hat{X}_{o}, \hat{Y}_{o}\right) \geq 1.5$ and explains the meaning of the first inequality in (15).

A similar explanation can be given to the second inequality in (15). Example 4 provides a further illustration of Theorem 2 .

Now consider the case of convex technologies. (As in the case of embedded technologies in Section 3, it suffices to assume that only technology $\hat{\mathcal{T}}$ is convex.) By inequalities (15) and the inequality $(4)$ restated for $\operatorname{DMU}\left(\hat{X}_{o}, \hat{Y}_{o}\right)$, we have

$$
\varepsilon^{+}\left(X_{o}, Y_{o}\right) \leq \hat{\varepsilon}^{+}\left(\hat{X}_{o}, \hat{Y}_{o}\right) \leq \hat{\varepsilon}^{-}\left(\hat{X}_{o}, \hat{Y}_{o}\right) \leq \varepsilon^{-}\left(X_{o}, Y_{o}\right)
$$

or, alternatively,

$$
\left[\hat{\varepsilon}^{+}\left(\hat{X}_{o}, \hat{Y}_{o}\right), \hat{\varepsilon}^{-}\left(\hat{X}_{o}, \hat{Y}_{o}\right)\right] \subseteq\left[\varepsilon^{+}\left(X_{o}, Y_{o}\right), \varepsilon^{-}\left(X_{o}, Y_{o}\right)\right]
$$

Similar to Section 3, it is worth restating the above results in terms of the RTS characterization. We first consider the case in which the RTS type of $\mathrm{DMU}_{o}$ has already been evaluated in technology $\mathcal{T}$.

Corollary 4. Let technology $\hat{\mathcal{T}}$ be convex and $D M U\left(\hat{X}_{o}, \hat{Y}_{o}\right) \in \hat{\mathcal{T}}$ satisfy Assumption 1 in technology $\hat{\mathcal{T}}$. Then

(i) If DMU $U_{o}$ exhibits IRS in technology $\mathcal{T}$, then $\widehat{\mathrm{DMU}}_{o}$ exhibits IRS in the aggregated technology $\hat{\mathcal{T}}$.

(ii) If $D M U_{o}$ exhibits DRS in technology $\mathcal{T}$, then $\widehat{\mathrm{DMU}}_{o}$ exhibits DRS in the aggregated technology $\hat{\mathcal{T}}$.

As in the case of embedded technologies considered in Section 3, if $\mathrm{DMU}_{o}$ exhibits CRS in technology $\mathcal{T}$, it may generally exhibit any of the three types of RTS in the aggregated technology $\hat{\mathcal{T}} .^{15}$

Assuming the opposite scenario in which we have already evaluated the RTS type of $\widehat{\mathrm{DMU}}_{o}$ in the aggregated technology $\hat{\mathcal{T}}$, we have the following implications for the RTS type of $\mathrm{DMU}_{o}$ in technology $\mathcal{T}$.

Corollary 5. Let technology $\hat{\mathcal{T}}$ be convex and $D M U\left(\hat{X}_{o}, \hat{Y}_{o}\right) \in \hat{\mathcal{T}}$ satisfy Assumption 1 in technology $\hat{\mathcal{T}}$. Then

(i) If $\widehat{\mathrm{DMU}}_{o}$ exhibits CRS in technology $\hat{\mathcal{T}}$, then DMU exhibits $C R S$ in technology $\mathcal{T}$.

\footnotetext{
${ }^{15}$ Similar to the case of embedded technologies discussed in Footnote 7, it is straightforward to show that, under the conditions of Corollaries 4 and 5, Definition 2 provides a complete characterization of RTS at the $\operatorname{DMU}\left(X_{o}, Y_{o}\right)$, even if technology $\mathcal{T}$ is not convex.
} 
Table 3: DMUs in Example 4.

\begin{tabular}{cccc}
\hline DMU & Input $x_{1}$ & Input $x_{2}$ & Output $y$ \\
\hline$A$ & 2 & 1 & 0.5 \\
$B$ & 6 & 2 & 2 \\
$C$ & 1 & 4 & 3 \\
\hline
\end{tabular}

(ii) If $\widehat{\mathrm{DMU}}_{o}$ exhibits IRS in technology $\hat{\mathcal{T}}$, then $D M U_{o}$ exhibits either IRS or CRS in technology $\mathcal{T}$ but cannot exhibit DRS

(iii) If $\widehat{\mathrm{DMU}}_{o}$ exhibits DRS in technology $\hat{\mathcal{T}}$, then $D M U_{o}$ exhibits either DRS or CRS in technology $\mathcal{T}$ but cannot exhibit IRS.

We can now make an observation similar to the case of embedded technologies considered in Section 3. Namely, as follows from formula (17), even if $\mathrm{DMU}_{o}$ in technology $\mathcal{T}$ retains its RTS type in the aggregated technology $\hat{\mathcal{T}}$, the range between the right-hand and left-hand scale elasticities in the latter technology is generally narrower than in the former. This means that the strength of the RTS characterization defined by this range is determined more precisely in the aggregated technology $\hat{\mathcal{T}}$ than in $\mathcal{T}$.

Remark 5. In the case of technologies with smooth production frontiers, the relationships established by Theorem 2 and its corollaries become more precise. Namely, under the conditions of this theorem, let $\varepsilon\left(X_{o}, Y_{o}\right)$ and $\hat{\varepsilon}\left(\hat{X}_{o}, \hat{Y}_{o}\right)$ be the scale elasticities evaluated at the $\mathrm{DMU}_{o}$ and $\widehat{\mathrm{DMU}}_{o}$, respectively. Then

$$
\varepsilon\left(X_{o}, Y_{o}\right)=\hat{\varepsilon}\left(\hat{X}_{o}, \hat{Y}_{o}\right) .
$$

This last equality implies that the type of RTS exhibited by a DMU in technology $\mathcal{T}$ remains the same in the technology obtained from $\mathcal{T}$ by aggregation or removal of some of its inputs and outputs (provided this DMU is output radial efficient in both technologies).

Example 4. Consider the VRS technology $\mathcal{T}_{\text {VRS }}$ with two inputs $x_{1}$ and $x_{2}$ and one output $y$ generated by the three observed DMUs $A, B$ and $C$ given in Table 3 . This technology is shown in Figure 4 as the area below the facets $A B C, K C L, L C B M$ and $M B A N$. Note that all three DMUs $A, B$ and $C$ satisfy Assumption 1.

The one-sided scale elasticities $\varepsilon^{+}$and $\varepsilon^{-}$at each of the observed DMUs in technology $\mathcal{T}_{\text {VRS }}$ have been computed using the standard linear programming approach ${ }^{16}$ and are shown in the second and third columns of Table 4.

Now suppose that we have omitted input $x_{1}$ and wish to evaluate the efficiency and RTS types in the resulting technology $\hat{\mathcal{T}}_{\text {VRS }}$ with the remaining single input $x_{2}$ and output $y$. Formally, we can view this technology as obtained by the aggregation of inputs $x_{1}$ and $x_{2}$ in technology $\mathcal{T}_{\text {VRS }}$ with the weights $a_{1}=0$ and $a_{2}=1$, respectively. By Proposition 1 , technology $\hat{\mathcal{T}}_{\text {VRS }}$ is the standard VRS technology in the single input and single output space generated by the three observed DMUs in Table 3 whose input $x_{1}$ is ignored. The resulting

\footnotetext{
${ }^{16}$ For each observed DMU, the right-hand and left-hand scale elasticities are found as $1-\omega_{\max }$ and $1-\omega_{\min }$, respectively, where $\omega_{\max }$ and $\omega_{\min }$ are the maximum and minimum values of the sign-free variable in the output-oriented multiplier VRS model, found by solving an appropriately specified linear program (see, e.g., Førsund \& Hjalmarsson, 2004).
} 


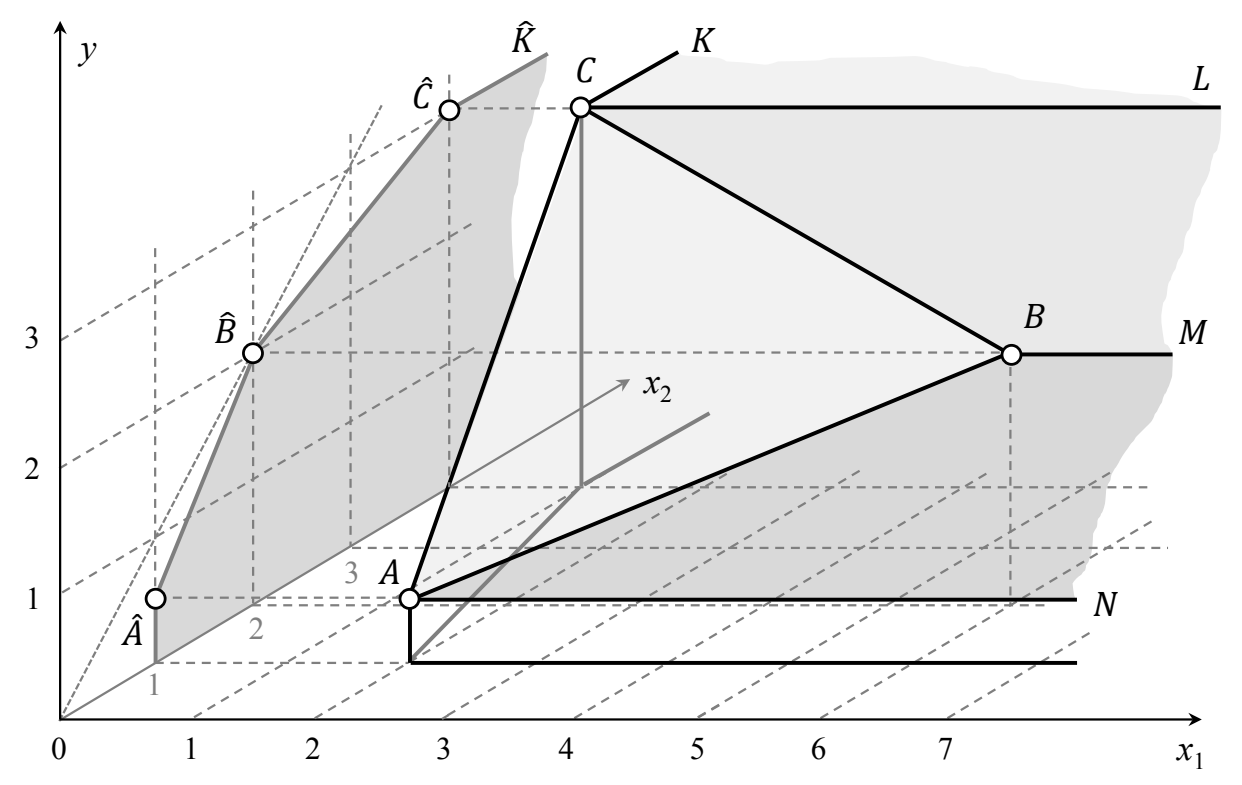

Figure 4: Technologies $\mathcal{T}_{\text {VRS }}$ and $\hat{\mathcal{T}}_{\text {VRS }}$ in Example 4.

Table 4: One-sided scale elasticities in Example 4.

\begin{tabular}{ccccc}
\hline & \multicolumn{2}{c}{ Technology } & $\mathcal{T}_{\text {VRS }}$ & \multicolumn{2}{c}{ Technology } & $\hat{\mathcal{T}}_{\text {VRS }}$ \\
\cline { 2 - 5 } $\mathrm{DMU}$ & $\varepsilon^{+}$ & $\varepsilon^{-}$ & $\hat{\varepsilon}^{+}$ & $\hat{\varepsilon}^{-}$ \\
\hline$A(\hat{A})$ & 2.38 & $+\infty$ & 3 & $+\infty$ \\
$B(\hat{B})$ & 0.5 & 1.5 & 0.5 & 1.5 \\
$C(\hat{C})$ & 0 & $+\infty$ & 0 & 0.67 \\
\hline
\end{tabular}

DMUs are denoted $\hat{A}, \hat{B}$ and $\hat{C}$ and plotted on the plane spanning the axes of the input $x_{2}$ and output $y$ in Figure 4 . The technology $\hat{\mathcal{T}}_{\text {VRS }}$ is shown as the shaded area below the line $\hat{A} \hat{B} \hat{C} \hat{K}$ on the same plane.

The one-sided scale elasticities in technology $\hat{\mathcal{T}}_{\text {VRS }}$ are found by simple computations similar to those used in Example 1 and are shown in the last two columns of Table 4. Note that these scale elasticities are consistent with Theorem 2 and the relationships (16) and (17) based on it. Namely, the range between the right-hand and left-hand scale elasticities in the aggregated technology $\hat{\mathcal{T}}_{\text {VRS }}$ is never wider than the corresponding range in the original technology $\mathcal{T}_{\text {VRS }}$.

Furthermore, by Definition 2, both DMUs $A$ and $\hat{A}$ exhibit IRS, and both DMUs $B$ and $\hat{B}$ exhibit CRS, in the corresponding technologies. However, DMU $C$ exhibits CRS in the original technology $\mathcal{T}_{\text {VRS }}$ but its aggregated variant $\hat{C}$ exhibits DRS in technology $\hat{\mathcal{T}}_{\text {VRS }}$. These findings illustrate the theoretical results of Corollaries 4 and 5 .

\section{Conclusion}

In this paper, we address the following question: how does the specification of production technology affect the RTS characterization of its efficient frontier? We consider two 
particular scenarios. In the first scenario we assume that our current model of technology is enlarged by the incorporation of additional DMUs or by any other information about the production process such as the specification of weight restrictions or changing the assumption of joint weak input disposability to their strong disposability. In the second scenario we assume that some inputs or outputs are aggregated or omitted, which results in the technology restated in a reduced number of dimensions. Of course, the opposite scenarios are also possible, in which we start with a larger technology and make it smaller or increase the number of dimensions by disaggregating some inputs and outputs or specifying new ones.

The notion of RTS is based on the notion of scale elasticity which is defined for smooth production frontiers. If the production frontier is not smooth, the RTS characterization is defined based on the notion of one-sided (left-hand and right-hand) scale elasticities. The type of RTS exhibited by the $\mathrm{DMU}_{o}$ under the assessment depends on the range between the right-hand and left-hand scale elasticities.

In this paper, we prove that, if we enlarge the technology, aggregate or remove some of its inputs or outputs, the right-hand scale elasticity evaluated at the DMUs on the production frontier does not decrease and the left-hand scale elasticity does not increase. In the case of convex technologies, this means that the range between the right-hand and left-hand scale elasticities becomes tighter.

This proved relationship is easily restated in terms of RTS, especially for convex technologies. For example, if $\mathrm{DMU}_{o}$ exhibits IRS or DRS in the smaller technology, it exhibits the same RTS type in the larger technology, provided it remains output radial efficient in the latter. Moreover, the range defined by the one-sided scale elasticities is tighter (narrower) in the latter technology which means that the strength of the RTS characterization represented by such range is more precise in the larger technology. The same relationship holds in the case of aggregation and exclusion of inputs and outputs.

If we consider the opposite scenario in which we replace a larger technology by its subset or if we disaggregate some of the inputs or outputs, the range defined by the one-sided scale elasticities at $\mathrm{DMU}_{o}$ generally becomes wider. This means, for example, that if $\mathrm{DMU}_{o}$ exhibits IRS in the larger technology, then it may exhibit either IRS or CRS in the smaller technology but cannot exhibit DRS.

In summary, we can combine our results with the known results related to efficiency scores as follows: selecting a larger technology or reducing the number of inputs and outputs (either by aggregation or removal) leads to improved discrimination on efficiency and to more precisely defined RTS characterization of production frontiers.

Our results suggest several further research avenues left outside the scope of this paper. First, it should be worthwhile to reinterpret these results in terms of the cost and revenue functions (Färe \& Grosskopf, 1985; Färe et al., 1985) which arise from the aggregation of inputs and, respectively, outputs with known prices. Second, it should be unproblematic to extend our results to the notion of partial scale elasticity and the corresponding partial RTS types, explored in the DEA context by Podinovski and Førsund (2010) and Podinovski et al. (2016). Such partial characteristics represent a response of a subvector of outputs to marginal proportional changes of a subvector of inputs, which is useful, for example, when some inputs and outputs are exogenously fixed. The described scenario requires the introduction of a partial analogue of the output response function introduced in Section 2. A similar extension is possible for directional definitions of RTS in the sense explored by Balk et al. (2015), and to various marginal rates of substitution and transformation involving different inputs and outputs. The restatement of our main results and modification of their proofs for all these cases are straightforward. 


\section{Appendix A. Proofs}

Proof of Theorem 1. Let $\tilde{\beta}(\alpha)$ be the output response function (1) defined at DMU $\left(X_{o}, Y_{o}\right)$ in technology $\tilde{\mathcal{T}}$, i.e.,

$$
\tilde{\beta}(\alpha)=\max \left\{\beta \mid\left(\alpha X_{o}, \beta Y_{o}\right) \in \tilde{\mathcal{T}}, \beta \in \mathbb{R}\right\},
$$

and let $\tilde{\Gamma}$ be its domain. Because $\left(\alpha X_{o}, \beta Y_{o}\right) \in \mathcal{T}$ implies $\left(\alpha X_{o}, \beta Y_{o}\right) \in \tilde{\mathcal{T}}$, for any $\alpha$ and $\beta$, we have $\Gamma \subseteq \tilde{\Gamma}$. Because $\mathcal{T} \subseteq \tilde{\mathcal{T}}$, we have $\bar{\beta}(\alpha) \leq \tilde{\beta}(\alpha)$, for all $\alpha \in \Gamma \subseteq \tilde{\Gamma}$. By Assumption 1 , we have $\bar{\beta}(1)=\tilde{\beta}(1)=1$. Then

$$
\bar{\beta}_{+}^{\prime}(1)=\lim _{\alpha \downarrow 1} \frac{\bar{\beta}(\alpha)-\bar{\beta}(1)}{\alpha-1} \leq \lim _{\alpha \downarrow 1} \frac{\tilde{\beta}(\alpha)-\tilde{\beta}(1)}{\alpha-1}=\tilde{\beta}_{+}^{\prime}(1) .
$$

The proof of the second inequality in (6) is similar.

Proof of Proposition 1. Denote $\bar{X}$ and $\bar{Y}$ the $m \times n$ and $s \times n$ matrices whose columns are the input and output vectors of observed DMUs $\left(X_{j}, Y_{j}\right), j=1, \ldots, n$, respectively. Denote $\bar{X}_{i}$ the row $i$ of matrix $\bar{X}$ and $\bar{Y}_{r}$ the row $r$ of matrix $\bar{Y}$. Taking into account (10), technology $\mathcal{T}_{\text {VRs }}$ is defined as the set of all DMUs $(X, Y) \in \mathbb{R}_{+}^{m} \times \mathbb{R}_{+}^{s}$ for which there exists a vector $\lambda \in \mathbb{R}^{n}$ such that

$$
\begin{aligned}
& \bar{X}_{i} \lambda \leq x_{i}, \quad i=1, \ldots, m, \\
& \bar{Y}_{r} \lambda \geq y_{r}, \quad r=1, \ldots, s, \\
& 1^{\top} \lambda=1, \quad \lambda \geq 0 .
\end{aligned}
$$

Technology $\hat{\mathcal{T}}_{\text {VRS }}$ is obtained by aggregation (12) of technology $\mathcal{T}_{\text {VRS }}$. Define technology $\overline{\mathcal{T}}_{\text {VRS }}$ as the VRS technology generated by the set of aggregated observed DMUs $\left(\hat{X}_{j}, \hat{Y}_{j}\right)$, $j=1, \ldots, n$, i.e., as the set of all DMUs $(\hat{X}, \hat{Y}) \in \mathbb{R}_{+}^{m^{\prime}} \times \mathbb{R}_{+}^{s^{\prime}}$, where $\hat{X}=\left(\hat{x}_{1}, \ldots, \hat{x}_{m^{\prime}-1}, \hat{x}_{m^{\prime}}\right)$ and $\hat{Y}=\left(\hat{y}_{1}, \ldots, \hat{y}_{s^{\prime}-1}, \hat{y}_{s^{\prime}}\right)$, for which there exists a vector $\mu \in \mathbb{R}^{n}$ such that:

$$
\begin{aligned}
& \bar{X}_{i} \mu \leq \hat{x}_{i}, \quad i=1, \ldots, m^{\prime}-1, \\
& \left(\sum_{i=m^{\prime}}^{m} \bar{X}_{i} a_{i}\right) \mu \leq \hat{x}_{m^{\prime}}, \\
& \bar{Y}_{r} \mu \geq \hat{y}_{r}, \quad r=1, \ldots, s^{\prime}-1, \\
& \left(\sum_{r=s^{\prime}}^{s} \bar{Y}_{r} b_{r}\right) \mu \geq \hat{y}_{s^{\prime}}, \\
& 1^{\top} \mu=1, \mu \geq 0 .
\end{aligned}
$$

We need to prove that $\hat{\mathcal{T}}_{\text {VRS }}=\overline{\mathcal{T}}_{\text {VRS }}$. Let $(\hat{X}, \hat{Y}) \in \hat{\mathcal{T}}_{\text {VRS }}$. By $(12),(\hat{X}, \hat{Y})$ is obtained by aggregation (11) from some $(X, Y) \in \mathcal{T}_{\text {VRS }}$, which satisfies (A.1) with some vector $\lambda^{\prime}$. Multiply each inequality $i=m^{\prime}, \ldots, m$ in (A.1a) by the corresponding $a_{i} \geq 0$ and add together, to obtain (A.2b) in which $\mu=\lambda^{\prime}$. Similarly, multiply each inequality $r=s^{\prime}, \ldots, s$ in (A.1b) by the corresponding $b_{r} \geq 0$ and add together, to obtain (A.2d) in which $\mu=\lambda^{\prime}$. Therefore, $(\hat{X}, \hat{Y})$ satisfies (A.2) with $\mu=\lambda^{\prime}$. This implies that $(\hat{X}, \hat{Y}) \in \overline{\mathcal{T}}$ and $\hat{\mathcal{T}}_{\text {VRS }} \subseteq$ $\overline{\mathcal{T}}_{\text {VRS }}$.

Conversely, let $(\hat{X}, \hat{Y}) \in \overline{\mathcal{T}}_{\text {VRS }}$, where $\hat{X}=\left(\hat{x}_{1}, \ldots, \hat{x}_{m^{\prime}-1}, \hat{x}_{m^{\prime}}\right)$ and $\hat{Y}=\left(\hat{y}_{1}, \ldots, \hat{y}_{s^{\prime}-1}, \hat{y}_{s^{\prime}}\right)$. Then DMU $(\hat{X}, \hat{Y})$ satisfies (A.2) with some $\mu^{\prime}$. We need to prove that there exists a DMU 
$\left(X^{*}, Y^{*}\right) \in \mathcal{T}_{\text {VRS }}$, where $X^{*}=\left(x_{1}^{*}, \ldots, x_{m}^{*}\right)$ and $Y^{*}=\left(y_{1}^{*}, \ldots, y_{s}^{*}\right)$, such that $(\hat{X}, \hat{Y})$ is obtained from $\left(X^{*}, Y^{*}\right)$ by the aggregation formula (11). Below we construct the required $\operatorname{DMU}\left(X^{*}, Y^{*}\right)$ which satisfies (A.1) with $\lambda=\mu^{\prime}$.

First, for all $i=1, \ldots, m^{\prime}-1$, let $x_{i}^{*}=\hat{x}_{i}$. Similarly, for all $r=1, \ldots, s^{\prime}-1$, let $y_{r}^{*}=\hat{y}_{r}$. This satisfies the first $m^{\prime}-1$ inequalities (A.1a) and the first $s^{\prime}-1$ inequalities (A.1b), with $\lambda=\mu^{\prime}$.

Next, we need to define components $x_{i}^{*}$ for $i=m^{\prime}, \ldots, m$, and $y_{r}^{*}$ for $r=s^{\prime}, \ldots, s$. Let $\mathcal{A}_{+}$ and $\mathcal{A}_{0}$ be the sets of all $i=m^{\prime}, \ldots, m$ such that $a_{i}>0$ and $a_{i}=0$, respectively. Similarly, let $\mathcal{B}_{+}$and $\mathcal{B}_{0}$ be the sets of all $r=s^{\prime}, \ldots, s$ such that $b_{r}>0$ and $b_{r}=0$, respectively.

For each $i \in \mathcal{A}_{0}$, define $x_{i}^{*}=\bar{X}_{i} \mu^{\prime} \geq 0$ and, for each $r \in \mathcal{B}_{0}$, define $y_{r}^{*}=\bar{Y}_{r} \mu^{\prime} \geq 0$. Such definitions, together with $\lambda=\mu^{\prime}$, satisfy the corresponding inequalities in (A.1a) and (A.1b) as equalities.

Now consider $i \in \mathcal{A}_{+}$and $r \in \mathcal{B}_{+}$. Define

$$
\bar{\alpha}=\left(\sum_{i \in \mathcal{A}_{+}} \bar{X}_{i} a_{i}\right) \mu^{\prime}, \quad \bar{\beta}=\left(\sum_{r \in \mathcal{B}_{+}} \bar{Y}_{r} b_{r}\right) \mu^{\prime} .
$$

If $\bar{\alpha}>0$, we multiply (A.2b) by $\alpha_{i}=\left(\bar{X}_{i} \mu^{\prime}\right) / \bar{\alpha}$, separately for each $i \in \mathcal{A}_{+}$, and define $x_{i}^{*}=\alpha_{i} \hat{x}_{m^{\prime}}$. Then the following inequality is true:

$$
\bar{X}_{i} \mu^{\prime} \leq x_{i}^{*}, \quad \forall i \in \mathcal{A}_{+} .
$$

If $\bar{\alpha}=0$, then $\bar{X}_{i} \mu^{\prime}=0$, for all $i \in \mathcal{A}_{+}$. In this case, define $x_{i}^{*}=\hat{x}_{m^{\prime}} / \sum_{i \in \mathcal{A}_{+}} a_{i}$. Then we have

$$
\bar{X}_{i} \mu^{\prime}=0 \leq x_{i}^{*}, \quad \forall i \in \mathcal{A}_{+} .
$$

The above completes the definition of the vector $X^{*}$. As shown, it satisfies all inequalities in (A.1a) with the vector $\lambda=\mu^{\prime}$. It is straightforward to verify that, in both cases $\bar{\alpha}>0$ and $\bar{\alpha}=0$, the aggregation of the vector $X^{*}$ by formula (11) produces vector $\hat{X}$.

Similarly, if $\bar{\beta}>0$, we multiply (A.2b) by $\beta_{r}=\left(\bar{Y}_{r} \mu^{\prime}\right) / \bar{\beta}$, separately for each $r \in \mathcal{B}_{+}$, and define $y_{r}^{*}=\beta_{r} \hat{y}_{s^{\prime}}$. Then we have

$$
\bar{Y}_{r} \mu^{\prime} \geq y_{r}^{*} \geq 0, \quad \forall r \in \mathcal{B}_{+} .
$$

If $\bar{\beta}=0$, then $\hat{y}_{s^{\prime}}=0$ and $\bar{Y}_{r} \mu^{\prime}=0$, for all $r \in \mathcal{B}_{+}$. Define $y_{r}^{*}=0$, for all $r \in \mathcal{B}_{+}$. Then

$$
\bar{Y}_{r} \mu^{\prime}=0=y_{r}^{*}, \quad \forall r \in \mathcal{B}_{+} .
$$

This completes the definition of the vector $Y^{*}$. As shown, it satisfies all inequalities in (A.1b) with $\lambda=\mu^{\prime}$. It is easy to verify that, in both cases $\bar{\beta}>0$ and $\bar{\beta}=0$, the aggregation of the vector $Y^{*}$ by formula (11) produces vector $\hat{Y}$.

In summary, we have defined DMU $\left(X^{*}, Y^{*}\right)$ which satisfies (A.1) with $\lambda=\mu^{\prime}$. Therefore $\left(X^{*}, Y^{*}\right) \in \mathcal{T}_{\text {VRS }}$. It is straightforward to verify that the aggregation of $\left(X^{*}, Y^{*}\right)$ using formula (11) produces DMU $(\hat{X}, \hat{Y})$. Therefore, $(\hat{X}, \hat{Y}) \in \hat{\mathcal{T}}_{\text {VRS }}$ and $\overline{\mathcal{T}}_{\text {VRS }} \subseteq \hat{\mathcal{T}}_{\text {VRS }}$. Taking into account the opposite embedding, we have $\hat{\mathcal{T}}_{\text {VRS }}=\overline{\mathcal{T}}_{\text {VRS }}$.

Proof of Proposition 2. Let $\beta^{\prime}$ be feasible in program (13), i.e., $\left(X_{o}, \beta^{\prime} Y_{o}\right) \in \mathcal{T}$. Then $\left(\hat{X}_{o}, \beta^{\prime} \hat{Y}_{o}\right)$ obtained by formula $(11)$ is in $\hat{\mathcal{T}}$. Therefore, $\beta^{\prime}$ is feasible in program (14), and the proof follows.

Proof of Corollary 3 . Let $\widehat{\mathrm{DMU}}_{o}$ satisfy Assumption 1 in technology $\hat{\mathcal{T}}$. Then, in program (14), $\hat{\beta}^{*}=1$. By Proposition 2 , in program (13), $\beta^{*} \leq 1$. Because $\beta=1$ is feasible in (13), we have $\beta^{*}=1$. Program (13) is the same as program (1). Therefore, DMU satisfies Assumption 1 in technology $\mathcal{T}$. 
Proof of Theorem 2. Let $\hat{\beta}(\alpha)$ be the output response function (1) defined at DMU $\left(\hat{X}_{o}, \hat{Y}_{o}\right)$ in technology $\hat{\mathcal{T}}$, i.e.,

$$
\hat{\beta}(\alpha)=\max \left\{\beta \mid\left(\alpha \hat{X}_{o}, \beta \hat{Y}_{o}\right) \in \hat{\mathcal{T}}, \beta \in \mathbb{R}\right\},
$$

and let $\hat{\Gamma}$ be its domain. For any $\alpha$ and $\beta,\left(\alpha X_{o}, \beta Y_{o}\right) \in \mathcal{T}$ implies $\left(\alpha \hat{X}_{o}, \beta \hat{Y}_{o}\right) \in \hat{\mathcal{T}}$. Therefore, $\Gamma \subseteq \hat{\Gamma}$ and $\bar{\beta}(\alpha) \leq \hat{\beta}(\alpha)$, for all $\alpha \in \Gamma \subseteq \hat{\Gamma}$. As assumed, we also have $\bar{\beta}(1)=\hat{\beta}(1)$. The rest of the proof is similar to the proof of Theorem 1 and is omitted.

Proof of Corollaries 1, 2, 4 and 5 . The proofs of all statements of these corollaries are similar. As an example, let us prove statement (i) of Corollary 1. Let DMU $\mathrm{D}_{o}$ exhibit IRS in $\mathcal{T}$. By Definition 2, we have $1<\varepsilon^{+}\left(X_{o}, Y_{o}\right) \leq \varepsilon^{-}\left(X_{o}, Y_{o}\right)$. Taking into account (7), we have $1 \leq \tilde{\varepsilon}^{+}\left(X_{o}, Y_{o}\right) \leq \tilde{\varepsilon}^{-}\left(X_{o}, Y_{o}\right)$. By Definition 2, DMU exhibits IRS in $\tilde{\mathcal{T}}$. 


\section{References}

Afriat, S. N. (1972). Efficiency estimation of production functions. International Economic Review, 13(3), $568-598$.

Ahn, T., Charnes, A., \& Cooper, W. W. (1988). Using data envelopment analysis to measure the efficiency of not-for-profit organizations: A critical evaluation-Comment. Managerial and Decision Economics, $9(3), 251-253$.

Allen, R., Athanassopoulos, A., Dyson, R. G., \& Thanassoulis, E. (1997). Weights restrictions and value judgements in data envelopment analysis: Evolution, development and future directions. Annals of Operations Research, 73, 13-34.

Balk, B. M., Färe, R., \& Karagiannis, G. (2015). On directional scale elasticities. Journal of Productivity Analysis, 43(1), 99-104.

Banker, R. D. (1984). Estimating most productive scale size using data envelopment analysis. European Journal of Operational Research, 17(1), 35-44.

Banker, R. D., Charnes, A., \& Cooper, W. W. (1984). Some models for estimating technical and scale efficiencies in data envelopment analysis. Management Science, 30(9), 1078-1092.

Banker, R. D., Cooper, W. W., Seiford, L. M., \& Zhu, J. (2011). Returns to scale in DEA. In W. W. Cooper, L. M, Seiford, \& J. Zhu (Eds.), Handbook on data envelopment analysis. (2nd ed.). (pp. 41-70). New York: Springer Science + Busines Media.

Banker, R. D., \& Thrall, R. M. (1992). Estimation of returns to scale using data envelopment analysis. European Journal of Operational Research, 62(1), 74-84.

Blackorby, C., \& Russell, R. R. (1999). Aggregation of efficiency indices. Journal of Productivity Analysis, 12(1), 5-20.

Boussofiane, A., Dyson, R. G., \& Thanassoulis, E. (1991) Applied data envelopment analysis. European Journal of Operational Research, 52(1), 1-15.

Briec, W., Dervaux, B., \& Leleu, H. (2003). Aggregation of directional distance functions and industrial efficiency. Journal of Economics, 79(3), 237-261.

Cesaroni, G., Kerstens, K., \& Van de Woestyne, I. (2017) Global and local scale characteristics in convex and nonconvex nonparametric technologies: A first empirical exploration. European Journal of Operational Research, 259(2), 576-586.

Chambers, R. G., Chung, Y., \& Färe, R. (1998). Profit, directional distance functions, and Nerlovian efficiency. Journal of Optimization Theory and Applications, 98(2), 351-364.

Chambers, R. G., \& Färe, R. (2008). A "calculus" for data envelopment analysis. Journal of Productivity Analysis, 30(3), 169-175.

Charnes, A., Cooper, W. W., \& Rhodes, E. (1978). Measuring the efficiency of decision making units. European Journal of Operational Research, 2(6), 429-444.

Cooper, W. W., Pastor, J. T., Borras, F., Aparicio, J., \& Pastor, D. (2011). BAM: A bounded adjusted measure of efficiency for use with bounded additive models Journal of Productivity Analysis, 35(2), 85-94.

Cooper, W. W., Seiford, L. M., \& Tone, K. (2000). Data envelopment analysis. A comprehensive text with models, applications, references and DEA-Solver software. New York: Springer Science + Business Media.

Deprins, D., Simar, L., \& Tulkens, H. (1984). Measuring labor-efficiency in post offices. In M. Marchand, P. Pestieau, \& H. Tulkens (Eds.), The performance of public enterprises: concepts and measurements (pp. 243-267). Amsterdam: North-Holland.

Diewert, W. E., \& Mendoza M. N. F. (2007). The Le Chatelier principle in data envelopment analysis. In R. Färe, S. Grosskopf, \& D. Primont (Eds.), Aggregation, efficiency, and measurement, (pp. 63-82). New York: Springer Science + Busines Media.

Färe, R., \& Grosskopf, S. (1985). A nonparametric cost approach to scale efficiency. Scandinavian Journal of Economics, 87(4), 594-604.

Färe, R., Grosskopf, S., \& Logan, J. (1983). The relative efficiency of Illinois electric utilities. Resources and Energy, 5(4), 349-367.

Färe, R., Grosskopf, S., \& Lovell, C. A. K. (1985). The measurement of efficiency of production. Boston: Kluwer Academic Publishers.

Färe, R., Grosskopf, S., \& Zelenyuk, V. (2004). Aggregation bias and its bounds in measuring technical efficiency. Applied Economics Letters, $11(10), 657-660$.

Färe, R., \& Zelenyuk, V. (2002). Input aggregation and technical efficiency. Applied Economics Letters, $9(10), 635-636$.

Førsund, F. R. (1996). On the calculation of scale elasticity in DEA models. Journal of Productivity Analysis, $7(2-3), 283-302$. 
Førsund, F. R., \& Hjalmarsson, L. (2004). Calculating scale elasticity in DEA models. Journal of the Operational Research Society, 55(10), 1023-1038.

Frisch, R. (1965). Theory of production. Dordrecht: D. Reidel Publishing Company.

Fukuyama, H. (2000). Returns to scale and scale elasticity in data envelopment analysis. European Journal of Operational Research, 125(1), 93-112.

Hadjicostas, P., \& Soteriou, A. C. (2006). One-sided elasticities and technical efficiency in multi-output production: A theoretical framework. European Journal of Operational Research, 168(2), 425-449.

Hanoch, G. (1970). Homotheticity in joint production. Journal of Economic Theory, 2(4), 423-426.

Imanirad, R., Cook, W. D., \& Zhu, J. (2013). Partial input to output impacts in DEA: Production considerations and resource sharing among business subunits. Naval Research Logistics, 60 (3), 190-207.

Jahanshahloo, M., Lofti, F. H., \& Zohrehbandian, M. (2005). Notes on sensitivity and stability of the classifications of returns to scale in data envelopment analysis. Journal of Productivity Analysis, 23(3), 309-313.

Johnson, W. E. (1913) The pure theory of utility curves. Economic Journal, 23(92), 483-513.

Kao, C. (2017). Network data envelopment analysis: Foundations and extensions. New York: Springer Science + Busines Media.

Mehdiloo, M., \& Podinovski, V. V. (2019). Selective strong and weak disposability in efficiency analysis. European Journal of Operational Research, 276(3), 1154-1169.

Nunamaker, T. R. (1985). Using data envelopment analysis to measure the efficiency of non-profit organizations: A critical evaluation. Managerial and Decision Economics, 6(1), 50-58.

Olesen, O. B., \& Ruggiero, J. (2014). Maintaining the regular ultra passum law in data envelopment analysis. European Journal of Operational Research, 235 (3), 798-809.

Panzar, J. C., \& Willig, R. D. (1977). Economies of scale in multi-output production. The Quarterly Journal of Economics, $91(3), 481-491$.

Podinovski, V. V. (2004a). Efficiency and returns to scale on the "no free lunch" assumption only. Journal of Productivity Analysis, 22(3), 227-257.

Podinovski, V. V. (2004b). Local and global returns to scale in performance measurement. Journal of the Operational Research Society, 55(2), 170-178.

Podinovski, V. V. (2004c). Production trade-offs and weight restrictions in data envelopment analysis. Journal of the Operational Research Society, 55(12), 1311-1322.

Podinovski, V. V. (2017). Returns to scale in convex production technologies. European Journal of Operational Research, 258(3), 970-982.

Podinovski, V. V., \& Bouzdine-Chameeva, T. (2013). Weight restrictions and free production in data envelopment analysis. Operations Research, 61(2), 426-437.

Podinovski, V. V., Chambers, R. G., Atici, K. B., \& Deineko, I. D. (2016). Marginal values and returns to scale for nonparametric production frontiers. Operations Research, 64 (1), 236-250.

Podinovski, V. V., \& Førsund, F. R. (2010). Differential characteristics of efficient frontiers in data envelopment analysis. Operations Research, 58(6), 1743-1754.

Podinovski, V. V., Olesen, O. B., \& Sarrico, C. S. (2018). Nonparametric production technologies with multiple component processes. Operations Research, 66 (1), 282-300.

Podinovski, V. V., \& Thanassoulis, E. (2007). Improving discrimination in data envelopment analysis: Some practical suggestions. Journal of Productivity Analysis, 28(1-2), 117-126.

Roll, Y., Cook, W. D., \& Golany, B. (1991). Controlling factor weights in data envelopment analysis. IIE Transactions, 23(1), 2-9.

Sahoo, B. K., \& Tone, K. (2015). Scale elasticity in non-parametric DEA approach. In J. Zhu (Ed.), Data envelopment analysis: A handbook of models and methods (pp. 269-290). New York: Springer Science + Busines Media.

Sahoo, B. K., Zhu, J., Tone, K., \& Klemen, B. M. (2014). Decomposing technical efficiency and scale elasticity in two-stage network DEA. European Journal of Operational Research, 233(3), 584-594.

Seiford, L. M., \& Zhu, J. (1999). Sensitivity and stability of the classifications of returns to scale in data envelopment analysis. Journal of Productivity Analysis, 12(1), 55-75.

Seiford, L. M., \& Zhu, J. (2005). Notes on sensitivity and stability of the classifications of returns to scale in data envelopment analysis: A comment. Journal of Productivity Analysis, 23(3), 315-316.

Starrett, D. A. (1977). Measuring returns to scale in the aggregate, and the scale effect of public goods. Econometrica, 45(6), 1439-1455.

Tauer, L. W. (2001). Input aggregation and computed technical efficiency, Applied Economics Letters, 8(5), 295-297.

Tauer, L. W., \& Hanchar, J. J. (1995). Nonparametric technical efficiency with K firms, N inputs and M outputs: A simulation. Agricultural and Resource Economics Review, 24(2), 185-189. 
Thanassoulis, E., \& Allen, R. (1998). Simulating weights restrictions in data envelopment analysis by means of unobserved DMUs. Management Science, 44(4), 586-594.

Thanassoulis, E., Dyson, R. G., \& Foster, M. J. (1987). Relative efficiency assessments using data envelopment analysis: An application to data on rates departments. Journal of the Operational Research Society, $38(5), 397-411$.

Thomas, A. C., \& Tauer, L. W. (1994). Linear input aggregation bias in nonparametric technical efficiency measurement. Canadian Journal of Agricultural Economics, 42(1), 77-86.

Thrall, R. M. (1989). Classification transitions under expansion of inputs and outputs in data envelopment analysis. Managerial and Decision Economics, 10(2), 159-162.

Zelenyuk, V. (2013). A scale elasticity measure for directional distance function and its dual: Theory and DEA estimation. European Journal of Operational Research, 228(3), 592-600.

Zelenyuk, V. (2015). Aggregation of scale efficiency. European Journal of Operational Research, 240(1), 269-277.

Zelenyuk, V. (2019). Data envelopment analysis and business analytics: The big data challenges and some solutions. Centre for Efficiency and Productivity Analysis, University of Queensland. Working Paper WP07/2019. 